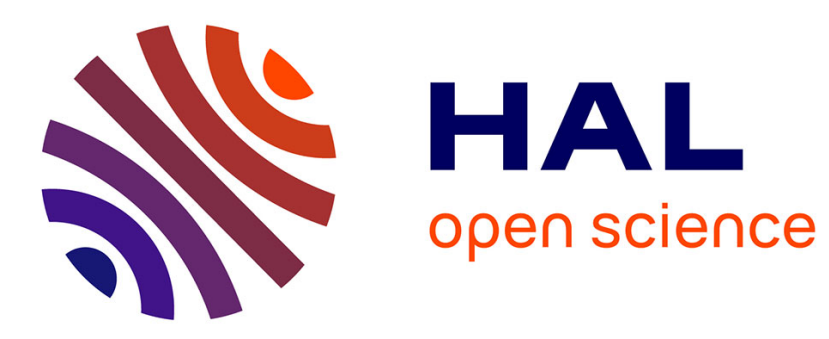

\title{
Rock Deformation Monitoring Using Monte Carlo Waveform Inversion
}

\author{
Ssu-Ting Lai, Nobuaki Fuji, Ikuo Katayama, Luis Fabian Bonilla, Yann
}

Capdeville

\section{- To cite this version:}

Ssu-Ting Lai, Nobuaki Fuji, Ikuo Katayama, Luis Fabian Bonilla, Yann Capdeville. Rock Deformation Monitoring Using Monte Carlo Waveform Inversion. Journal of Geophysical Research : Solid Earth, 2021, 126, 12 pp. 10.1029/2021JB021873 . insu-03589891

\section{HAL Id: insu-03589891 https://hal-insu.archives-ouvertes.fr/insu-03589891}

Submitted on 3 Mar 2022

HAL is a multi-disciplinary open access archive for the deposit and dissemination of scientific research documents, whether they are published or not. The documents may come from teaching and research institutions in France or abroad, or from public or private research centers.
L'archive ouverte pluridisciplinaire HAL, est destinée au dépôt et à la diffusion de documents scientifiques de niveau recherche, publiés ou non, émanant des établissements d'enseignement et de recherche français ou étrangers, des laboratoires publics ou privés.

$$
\text { Copyright }
$$




\section{JGR Solid Earth}

\section{RESEARCH ARTICLE}

10.1029/2021JB021873

Key Points:

- 3D numerical modeling with proper physical setting can simulate the waveforms in laboratory rock experiments

- Monte Carlo waveform inversion for velocity and attenuation structure can image velocity and attenuation changes during rock deformation

- Changes in velocity and attenuation show the different trends especially before rock failure

Correspondence to:

S.-T. Lai,

lai@ipgp.fr

Citation:

Lai, S.-T., Fuji, N., Katayama, I., Bonilla, L. F., \& Capdeville, Y. (2021). Rock deformation monitoring using Monte Carlo waveform inversion. Journal of Geophysical Research: Solid Earth, 126, e2021JB021873. https://doi. org/10.1029/2021JB021873

Received 15 FEB 2021 Accepted 9 SEP 2021

\section{Rock Deformation Monitoring Using Monte Carlo Waveform Inversion}

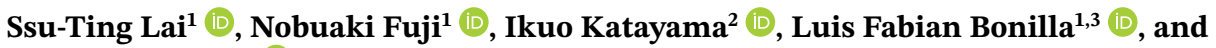 \\ Yann Capdeville ${ }^{4}$ (1)
}

${ }^{1}$ Université de Paris, Institut de physique du globe de Paris, CNRS, Paris, France, ${ }^{2}$ Department of Earth and Planetary Systems Science, Hiroshima University, Higashi-Hiroshima, Japan, ${ }^{3}$ Université Gustave Eiffel, Cité Descartes, Marne-laVallée, France, ${ }^{4}$ Laboratoire de Planétologie et de Géodynamique de Nantes, Nantes, France

\begin{abstract}
We estimate elastic and anelastic parameters and their evolution during laboratory rock deformation experiments, while developing a Monte Carlo waveform inversion. The transducer-totransducer one-source one-station active seismic data of dry and water-saturated samples are obtained from Zaima and Katayama (2018), https://doi.org/10.1029/2018JB016377. We first performed a trial-anderror estimate of the boundary conditions in order to suppress its influence on waveforms. The synthetic seismic data were generated using equivalent homogeneous models with different combinations of elastic and anelastic parameters with the aid of spectral element method. We compared them with the laboratory experimental data. Based on the comparisons, we obtained the time-lapse variations of seismic velocities and attenuation of rock samples during deformation experiments, which we interpreted as crack developments. Our simultaneous estimation of elastic and anelastic parameters allowed us to detail the dynamics prior to the rock failure.
\end{abstract}

Plain Language Summary We developed a novel methodology to monitor the rock deformation in the laboratory using active seismic waveforms, comparing them against 3D synthetic seismograms. Our methodology can invert for the P- and S-wave velocity changes as well as their attenuation quality factors. Changes in velocity and attenuation show the different trends especially just before rock failure, indicating the complexity of microcracks' evolution.

\section{Introduction}

In order to understand the earthquake nucleation, it is indispensable to study both macroscopic and microscopic behaviors of the rupture process of the Earth's crust and mantle: by global-scale numerical modeling (Fliss et al., 2005; Gabriel et al., 2012); natural earthquakes observation (Di Carli et al., 2010; Lee et al., 2011); and laboratory experiments of rock samples (Benson et al., 2006; Guéguen \& Schubnel, 2003; Lockner et al., 1977; Toksöz et al., 1979). Since averaged elastic wave velocities of a loaded rock sample could reflect the localized nucleation and/or growth of cracks, it is important to understand time-lapse changes of an experimental rock during its deformation to get insights on the macroscopic earthquake generation process (Scholz et al., 1973).

There have been several efforts to seismologically image the interior of rock samples. Möllhoff et al. (2010) simulated the preexisting artificial fracture in the rock using 2D discrete elastic lattice method, whereas Lai et al. (2019) studied the velocity model changes during rock deformation using the 2D finite differences method. However, 2D numerical modeling is insufficient to describe wave propagation in a 3D rock, even though the transfer function of Lai et al. (2019) or a 2D line source approximation (Igel et al., 2002) seems to be functioning to some extent. In order to surpass the limits, there have been some promising recent efforts of 3D numerical modeling of seismic waveforms in a laboratory scale, albeit a number of difficulties. Yoshimitsu et al. (2016), for instance, modeled the geometry effect on cylindrical aluminum using the 3D finite difference method, for a cylinder with $50 \mathrm{~mm}$ in diameter and $100 \mathrm{~mm}$ in height with a dominant frequency between 200 and $800 \mathrm{kHz}$. Their comparisons between synthetics and observed data, being limited to homogeneous metal and plastic experiments, have shown the feasibility of the numerical reproducibility of active seismic data in laboratory. In addition to wave propagation simulation, Brantut (2018) monitored acoustic emission on small-scaled sandstone using a 3D fast-marching method with finite differences and
(C) 2021. American Geophysical Union. All Rights Reserved. 
concomitantly constructed the evolution of velocity changes of the rock during its deformation. Sarout et al. (2017) estimated the evolution of crack parameters by inverting elastic waves and permeability data. Zotz-Wilson et al. (2020) imaged the development of fractures using migration of active seismic data, which they called coda wave decorrelation inversion. The presented studies are encouraging but some of the numerical treatments of the present complex problem should be fostered. The boundary condition employed in finite differences method is unrealistic (Komatitsch \& Vilotte, 1998), and the kernels used in the migration are too simplified using generalized ray theory. The aim of the present work is to extend the idea, combine with 3D numerical modeling on wave propagation, and carefully treat the entire waveforms in order to extract information on elastic and anelastic parameters changes.

Here, we would like to develop a methodology which inverts the seismic waveforms for structural changes during laboratory experiments performed by Zaima and Katayama (2018). They performed a triaxial compression experiment on the Aji granite and performed active seismic measurements during the rock deformation. The idea is to robustly and quantitatively infer the subtle structural changes of anelasticity as well as elasticity, and three-dimensional structure in the near future. There are little number of previous studies that attempted "waveform inversion" of laboratory experiments. We use the spectral element method (SEM: Komatitsch \& Vilotte, 1998; Chaljub et al., 2007) to model wave propagation in a rock sample during deformation. The spectral element method can accurately handle the boundary condition in 3D complex models (Capdeville et al., 2003; Chaljub et al., 2015; De Basabe \& Sen, 2014; Peter et al., 2011). However, due to its computational costs, there are only few studies devoted to the comparison of SEM synthetics with laboratory experimental data (e.g., Pageot et al., 2017; Solymosi et al., 2018). Here, we perform SEM modeling on one-source and one-receiver experimental data, in order to show the adaptability of SEM to the seismic data in laboratory. Throughout 3D simulation, we systematically construct a database of waveforms for different sets of elastic and anelastic parameters: P-wave velocity $\left(V_{P}\right)$, S-wave velocity $\left(V_{S}\right)$, P-wave quality factor $\left(Q_{P}\right)$ and S-wave quality factor $\left(Q_{S}\right)$. We then perform Monte Carlo waveform inversion using an objective function which combines three kinds of cost functions: $L_{1}$-norm and $L_{2}$-norm of waveform misfit and zero-lag cross correlation. We would like to explore information on rock elastic properties and attenuation changes using numerical simulation and Monte Carlo inversion. Throughout our approach, we will show how numerical models help to understand the observations and to illustrate seismic characteristics of rock deformation.

\section{Data and Methods}

In this study, we perform inversions to image elastic and anelastic structural changes during the rock deformation. Hence, we first acquire the active seismic data during the deformation experiment. Meanwhile, we prepare a set of synthetic seismograms with the aid of spectral element method. We then select the preferred models for observed seismograms (compressional and shear strains) for each differential stress step, in order to obtain the time-lapse trend of elastic and anelastic parameters. First, we revisit briefly the experiment configurations and provide known and unknown properties in the experiments. Second, we describe the numerical modeling scheme. Finally, we present our data processing strategy based on the data and modeling conditions and detail the inverse problem implemented in this study.

\subsection{Experimental Setup}

Triaxial compression experiments apply a confining pressure to a cylindrical rock sample wrapped in an impervious membrane, and then loads it axially to failure with dynamic compression. Zaima and Katayama (2018) conducted triaxial compression experiment on the Aji granite, which mainly contains quartz, plagioclase, $\mathrm{K}$-feldspar and biotite, with an average grain size of about $0.3 \mathrm{~mm}$ (Kudo et al., 1992). Both the dry and wet conditions were applied during the experiments. The wet experiment was under fluid-saturated condition, where distilled water was used as a pore fluid. The pore pressure of wet experiment was kept constant at $10 \mathrm{MPa}$ during the deformation. The experiment was performed at room temperature with a confining pressure of $20 \mathrm{MPa}$ and a constant strain rate of $1.3 \times 10^{-6} \mathrm{~s}^{-1}$.

The rock sample was roughly in the shape of a cylinder with a diameter of $20 \mathrm{~mm}$ and a height of $40 \mathrm{~mm}$. Two piezoelectric transducers were glued at two opposite sides of the rock sample, and were used as a 
(a)

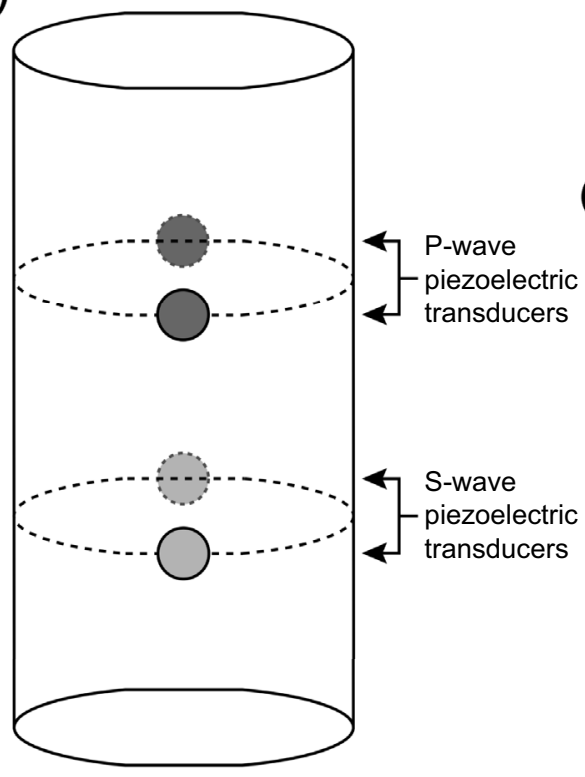

(b)

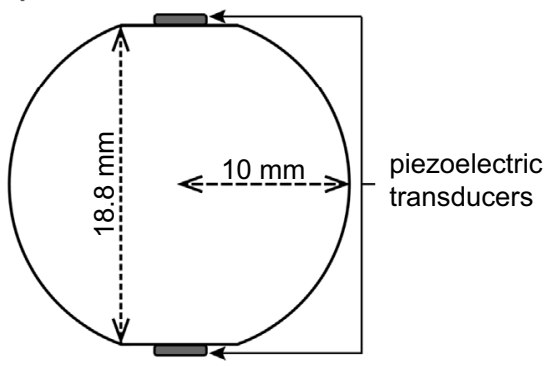

(c)

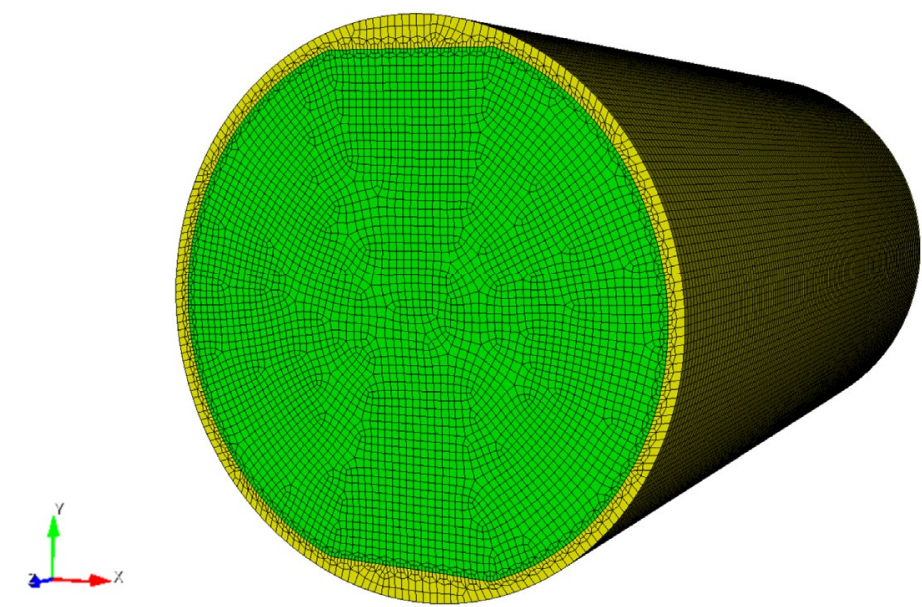

Figure 1. The schematic diagram of the geometry of sample, the position of the transducers and the numerical model used in this study. (a) The general view of the experimental setting of the sample. (b) The cross-section of the sample. (c) The distorted hexahedral mesh of the model for spectral element method. The green region is the rock model. The yellow part represents the surrounding low-velocity material. The mesh size is approximately $0.3 \mathrm{~mm}$.

source and a receiver, respectively. $\mathrm{P}$ waves and $\mathrm{S}$ waves were generated and recorded separately using piezoelectric transducers located at the symmetrical sides of the center point at the edge (Figure 1a). For the purpose of gluing the transducers, the two opposite sides of the sample were cut $0.6 \mathrm{~mm}$ off and polished, as shown in Figure 1b. Each P- and S-wave waveform with increasing differential stress was obtained by stacking signals for each compression condition during the rock deformation until the failure of the rock sample. When the compression stress approaches the critical state, which varies from one rock sample to another, the rock breaks. All the experiments data and details can be found in Zaima and Katayama (2018). 


\subsection{Forward Modeling of 3D Elastic Waves Using Spectral Element Method}

In our work, we use the spectral element code SEM3D (Cupillard et al., 2012; Delavaud, 2007) to compute synthetic seismograms traveling through the cylindrical rock samples. Here, we briefly review the spectral element method but the readers can refer to Komatitsch and Vilotte (1998) and Chaljub et al. (2007). The SEM is based upon a high-order polynomial approximation of the weak formulation of the wave equation. It combines the accuracy of the pseudospectral method with the flexibility of the finite element method. In this method, the wavefield is represented in terms of high-degree Lagrange interpolants per elements, and integrals are computed based upon the Gauss-Lobatto-Legendre (GLL) quadrature. Combined with a tensorial formulation, it leads to a perfectly diagonal mass matrix, which in turn leads to a fully explicit time scheme that is suitable to numerical simulations on parallel computers. The method allows a low dispersion error together with an accurate and implicit description of the boundary conditions. Nevertheless, the tensorial formulation imposes to use hexahedral meshes. We model cut and glued rock samples mimicking laboratory conditions as close as possible.

The Galerkin weak form of equation of motion (Geller \& Ohminato, 1994; Komatitsch \& Vilotte, 1998) reads:

$$
\left(\mathbf{T} \frac{\partial^{2}}{\partial t^{2}}+\mathbf{H}\right) \mathbf{u}(t)=\mathbf{g}(t),
$$

where $\mathbf{T}$ is mass matrix, the stiffness matrix $\mathbf{H}$ relates the elasticity and anelasticity, $\mathbf{g}$ represents the source term, and $\mathbf{u}$ is the discretized displacement for each element/point in the model. We use the free surface boundary (natural boundary condition) for the whole medium. However, in the reality, the rock sample was surrounded by a silicone jacket with high attenuation and slow velocity that could play a role as the partial-absorbing boundary with respect to the rock sample.

In order to model anelastic attenuation in our time-marching spectral element methods, SEM3D uses standard Zener linear solids (SLS) to approximate a nearly constant quality factor $Q$ for $\mathrm{P}$ and $\mathrm{S}$ waves (Carcione et al., 1988; Emmerich \& Korn, 1987; Liu et al., 1976; Moczo \& Kristek, 2005). A Zener body consists of a spring in series of the medium properties in connection with their relaxed stage at time $t=\infty$. SLS assumes that $\mathrm{Q}$ does not depend on frequency. In this study, we use three SLS on a band of relaxation frequency ranging from $33.3 \mathrm{kHz}$ to $3.3 \mathrm{MHz}$.

\subsubsection{Meshing}

In order to simulate realistic wave propagation, for the sake of seeking boundary conditions including silicone rubber over the rock, one needs to carefully mesh the medium in SEM computation. The standard GLL quadrature of SEM requires hexahedral meshes. However, it is known to be challenging to create such nonstructured hexahedral meshes with acceptable computational cost and high accuracy for a complex geometry. In this study, we used Trelis software (https://urldefense.com/v3/_https://coreform. com_;!!N11eV2iwtfs!6Ub8yKpVonc2RehbF-2k5BPhPC0B8mjBgvP9GS2FRSoHsV01CzzuiSmjvL04a2M\$) to mesh the model. In the case of nonstructured meshes, one has to avoid distorted elements, which may cause numerical instabilities.

Figure 1c shows the meshing used in our forward modeling. To avoid the numerical error caused by the contact between more than one medium, we must include a layer as refinement at the boundaries of different materials. Since we use polynomials of degree five as trial functions inside each element, the wavelength should be larger than the element size. Considering the minimum $\mathrm{S}$-wave velocity of $2,500 \mathrm{~m} / \mathrm{s}$ in the granite rock and the maximum target frequency of $2 \mathrm{MHz}$, the mesh size is set up to be approximately $0.3 \mathrm{~mm}$ in all models.

\subsubsection{Boundary Conditions}

The rock samples used in laboratory experiments are roughly cylindrical rocks. However, the sample was cut to form two parallel sharp surfaces, hence forming an incomplete cylinder. This imperfection of the cylindrical nature can cause a non-negligible effect on the waveforms. Although the rock sample itself is the main target of our simulation, a single-material approximation with free surface boundary would fail 
Table 1

The Description of Physical Parameter of Shin-Etsu KE45 W RTV Silicone Rubber (Properties: Silicone Rubber, 2020)

\begin{tabular}{lc}
\hline Physical parameter & Value \\
\hline Density & $1,050 \mathrm{~kg} / \mathrm{m}^{3}$ \\
Bulk modulus & $1.5-2 \mathrm{GPa}$ \\
Poisson's ratio & $0.47-0.49$ \\
Shear modulus & $0.3-20.0 \mathrm{MPa}$ \\
Young's modulus & $1.0-50.0 \mathrm{MPa}$ \\
\hline
\end{tabular}

in waveform modeling due to the surrounding materials. Materials such as silicone rubber, glue, aluminum frame, shrink plastic attached to the granitic rock may affect the waves propagating in the rock. Therefore, we incorporate complex boundary conditions to find the suitable model setting without losing computational efficiency. Furthermore, we investigate the boundary conditions with additional physical layer of Shin-Etsu KE45 W RTV silicone rubber (Table 1) to simulate the transducer waveforms and obtain the preferred model (Figure 1c). The comparison of the four models tested for boundary condition is described in Appendix A. The additional boundary conditions successfully mimicked quasi-surface waves generated at the rock-rubber discontinuity.

\subsubsection{Source and Transfer Functions}

In addition to the boundary setting and model geometry, the source time function and the source-receiver relative position will also cause a significant impact on the waveforms. To mimic the laboratory experiment, we set $\mathrm{P}$-wave source as an isotropic explosion source and S-wave source to be a shear stress along $\mathrm{X}-\mathrm{Z}$ direction. The source time function is formally taken from the experiment input waveform and then filtered as described in Appendix B.

During the experiments, compressional and shear waves are transmitted independently at different source positions and their strain waveforms are recorded at their corresponding antipodal points. In the reality, the transducers are glued to the rock sample. However, strains are not always continuous and we have less knowledge on coupling between the transducers, silicone rubber, and the rock sample. We thus consider four possible effective source-receiver distributions: (a) both the source and the receiver are slightly shifted inside the region of the rock model; (b) both the source and the receiver are slightly shifted to the region of the surrounding model; (c) the source is slightly shifted to the region of the surrounding model and the receiver is inside the region of the rock model; and (d) the receiver is slightly shifted to the region of the surrounding model and the source is inside the region of the rock model. The four source-receiver position scenarios are tested against the observed data for $\Delta \sigma=0$ (Figure 2). Compared to setting (b), the simulated waveforms of settings (a), (c) and (d) do not match the observed waveform. When the source and receiver are both located slightly outside the rock region (setting b), the first phases up to the fifth peak (4.0-6.2 $\mu \mathrm{s}$ ) have higher amplitudes than the other configurations. Therefore, we use the setting (b) as the source-receiver configuration in this study. We will discuss in detail the possible reasons for this preference in Section 4.

\subsection{Processing of Observed and Synthetic Data}

In the study, we aim to directly compare the observed and synthetic waveforms in order to infer the structural changes during the deformation experiment. By carefully treating 3D geometry, attenuation and boundary conditions as we discussed above, our method is advantageous to foster understanding of rock deformation compared to our previous studies (e.g., Lai et al., 2019). We had to introduce transfer functions to compensate unrealistic numerical settings such as $2 \mathrm{D}$ assumptions, pure-elastic approximations and free-surface boundary conditions.

According to the time-dependent spectrogram analysis in Figure 3, the strongest energy of the experimental waveform is concentrated in the frequency band between 1.6 and 3.0 $\mathrm{MHz}$ for P-wave records and in the frequency band between 1.0 and $2.8 \mathrm{MHz}$ for $\mathrm{S}$-wave. Compared to S-wave temporal spectrogram that indicates a constant dominant frequency range, P-wave spectrogram shows lower frequency energy $(\sim 500 \mathrm{kHz})$ after $7 \mu \mathrm{s}$ in addition to the most concentrated high-frequency region at the beginning. In this study, however, we focus on the variation in the high-frequency regime in order to capture the influence caused by inner structure changes. Two Butterworth bandpass filters with frequency ranges from 1.5 to $3.5 \mathrm{MHz}$ and from 1.0 to $3.0 \mathrm{MHz}$ are applied on the P- and S-wave data, respectively. A Hann window is then applied to data after filtering to extract the first arrivals. Note that we applied the same data processing treatments both to observed and synthetic waveforms. 
(a)

a)
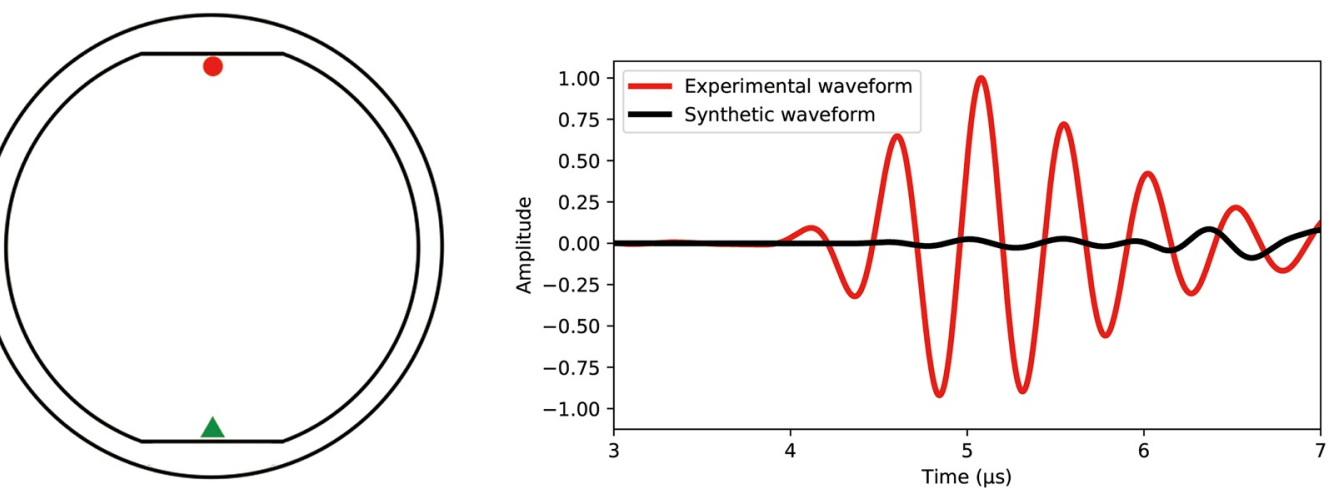

(b)
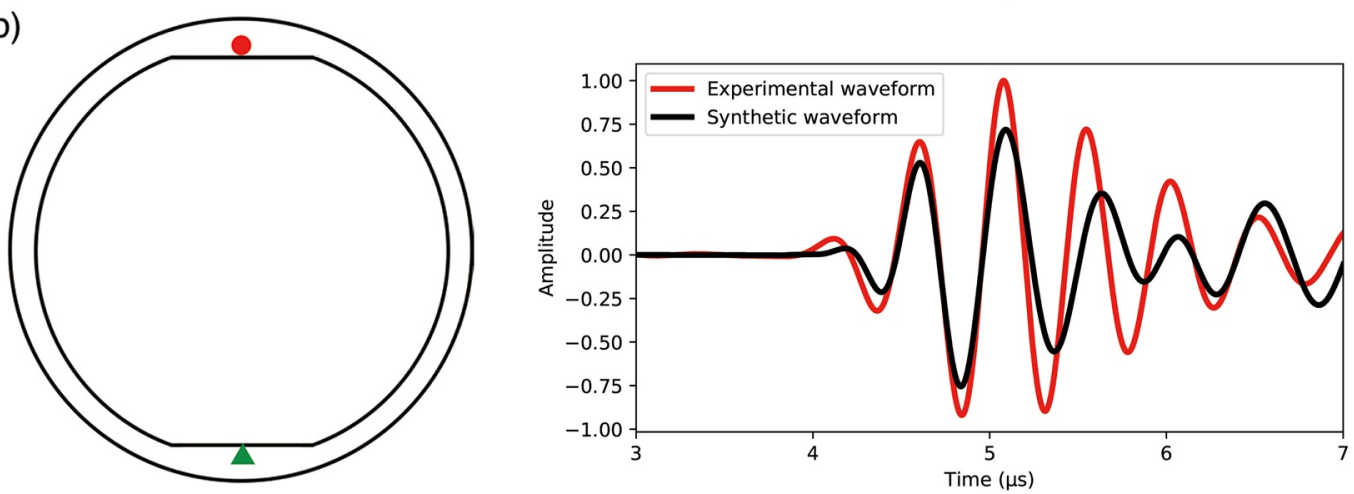

(c)
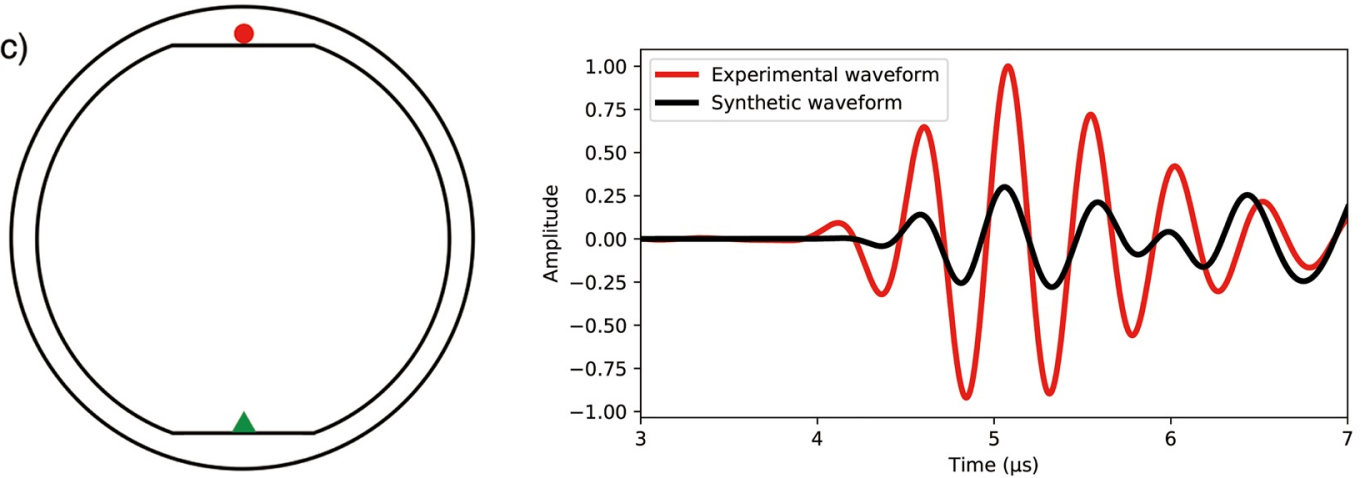

(d)
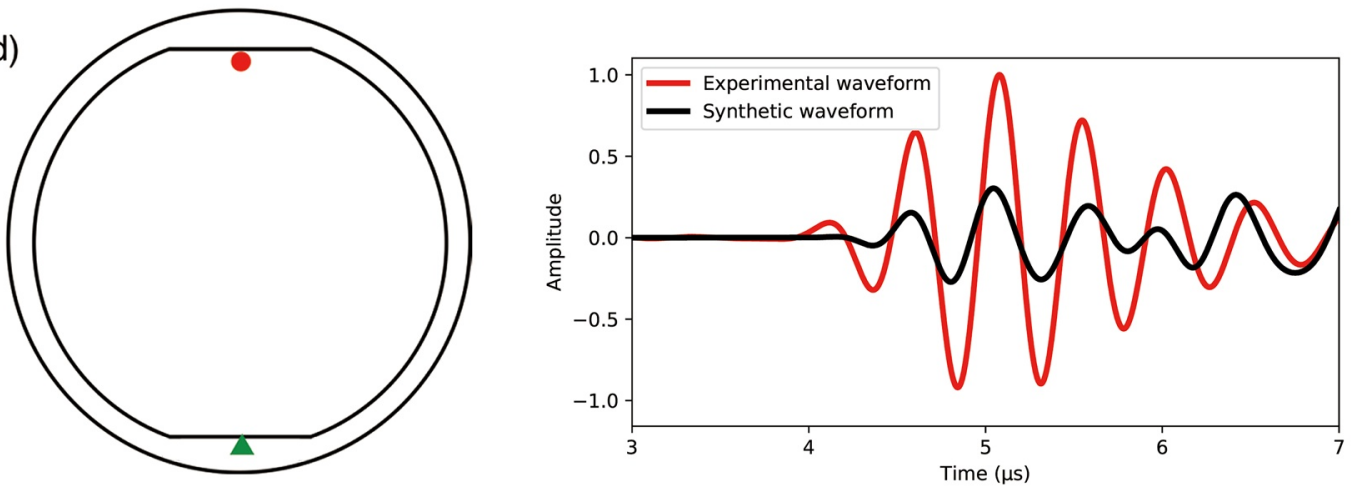

Figure 2. The four source-receiver positions tested in the study on the preferred model (Figure 1c) and their normalized-synthetic waveform compared to the data. The red circle represents the source position; contrariwise, the green triangle indicates the receiver position. The four source-receiver positions: (a) source and receiver are both sightly inside the inner material, (b) source and receiver are both slightly outside the inner material, (c) source is slightly outside but receiver is slightly inside the inner material, (d) source is slightly inside but receiver is slightly outside the material. The (b) setting of the source-receiver position is determined to be the best. 
(a)
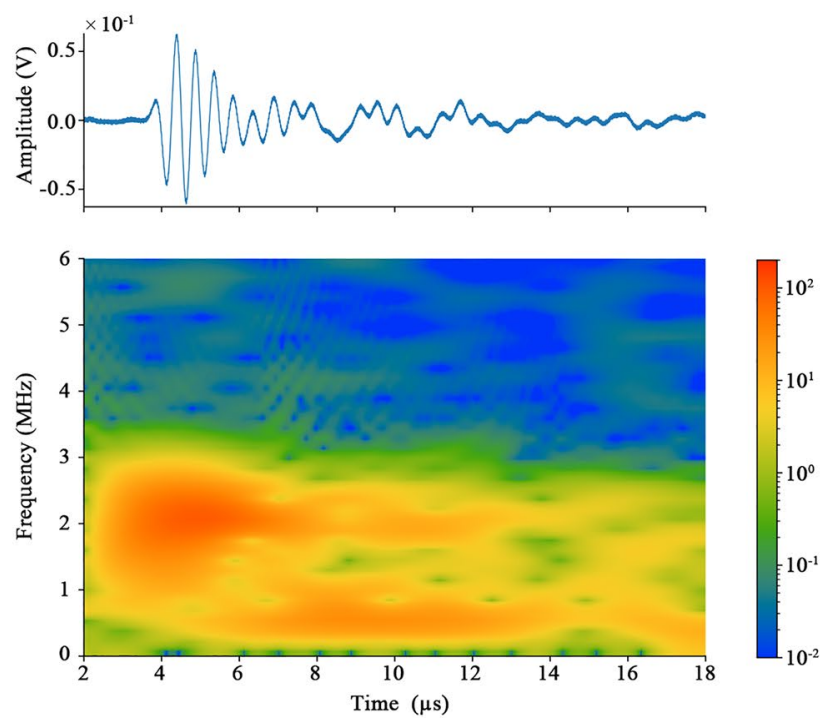

(b)
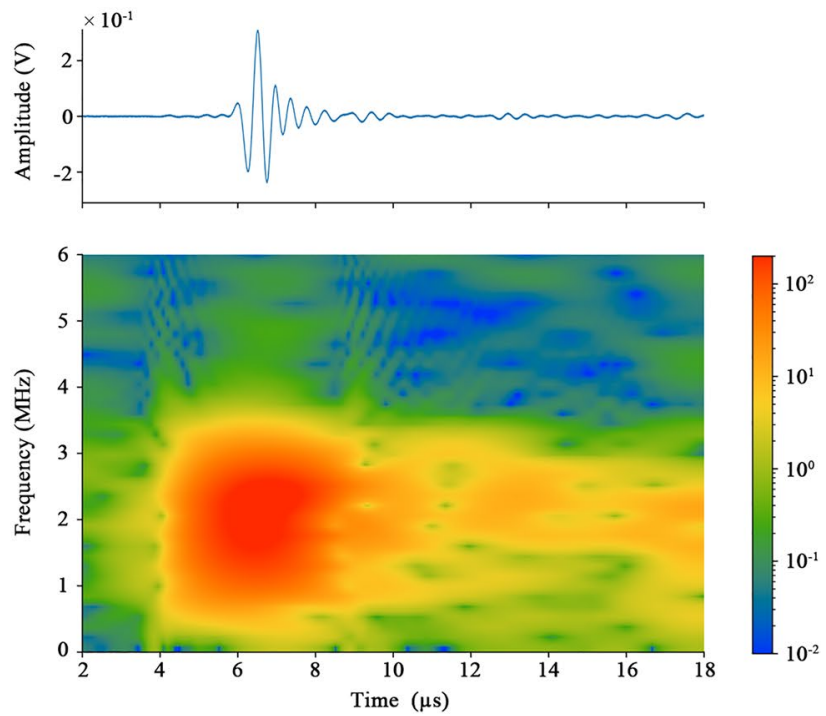

Figure 3. The (a) P- and (b) S-wave spectrogram of experimental data for 0 MPa differential stress.

\subsection{Inverse Problem}

We use waveforms for differential stress $\Delta \sigma$ :

$$
\mathbf{d}^{T}(\Delta \sigma)=\left[\mathbf{d}_{P P}^{T}(\Delta \sigma) \mathbf{d}_{S S}^{T}(\Delta \sigma)\right]
$$

where $\mathbf{d}_{P P}$ and $\mathbf{d}_{S S}$ are time series vectors of compressional and shear strain waveforms recorded at the antipodal receiver (Figure 1a). The superscript $T$ denotes transpose. We then generate synthetic seismograms $\mathbf{u}(\mathbf{m})$ with the same geometrical and source configurations for a set of seismological structural parameters m. We define a objective function to maximize:

$$
S(\mathbf{m}, \Delta \sigma)=\alpha \frac{\mathbf{d}^{T}(\Delta \sigma) \mathbf{u}(\mathbf{m})}{|\mathbf{d}(\Delta \sigma)||\mathbf{u}(\mathbf{m})|}+\beta \frac{1}{|\mathbf{d}(\Delta \sigma)-\mathbf{u}(\mathbf{m})|}+\gamma \frac{1}{|\mathbf{d}(\Delta \sigma)-\mathbf{u}(\mathbf{m})|^{2}}
$$

with $\alpha, \beta, \gamma$, the weighting factors for zero-lag cross correlation, reciprocals of $L_{1}$-norm and $L_{2}$-norm, respectively. First, Equation 3 is used to constrain the main phase with a large $\alpha$. Second, Equation 3 with large $\beta$ and $\gamma$ is used to investigate the amplitude of the waveform, and to further define the change in $Q$. The choice of $(\alpha, \beta, \gamma)$ depends on the data set since $\beta$ and $\gamma$ are difficult to fix from one experiment to another. This should be discussed more in the upcoming studies but here in this study we use our ad-hoc values as discussed in results section.

As the Aji granite is fine-grained and nearly isotropic, we can assume a homogeneous medium with a wavelength of 2-3 mm. We use four parameters to represent the model vector $\mathbf{m}^{T}=\left(V_{P}, V_{S}, Q_{P}, Q_{S}\right)$. This parameterization is to homogenize 3D an-/elastic heterogeneities. The time-lapse change of these global parameters will be related to the microscopic short-wavelength structural changes such as crack generation. The anelastic attenuation can be an indicator of the 3D elastic heterogeneity and intrinsic attenuation. Based on the a priori information on the Aji granite, we set $V_{P}$ varying from 3,700 to 5,800 m/s and $Q_{P}$ from 60 to $200\left(Q_{P}^{-1}\right.$ from $5.0 \times 10^{-3}$ to $\left.1.7 \times 10^{-2}\right)$ for the P-wave. As for the $S$-wave, $V_{S}$ varies from 2,660 to $3,550 \mathrm{~m} / \mathrm{s}$ and $Q_{S}$ from 20 to $90\left(Q_{S}^{-1}\right.$ from $1.1 \times 10^{-2}$ to $\left.5.0 \times 10^{-2}\right)$. Note that all the comparison is based on the waveform analysis for $\Delta \sigma=0$. We are interested in the relative evolution of the velocity and attenuation parameters to the initial status $\Delta \sigma$, which can be expressed as $\delta \mathbf{m}$, instead of the absolute values of $\mathbf{m} . \delta \mathbf{m}$ can thus give us the insight into the relative changes of the rock. 
Table 2

Setting Range of Elasticity Parameters and Attenuation Parameters

\begin{tabular}{lccccc}
\hline Model & $V_{P}(\mathrm{~m} / \mathrm{s})$ & $V_{S}(\mathrm{~m} / \mathrm{s})$ & Density $\left(\mathrm{kg} / \mathrm{m}^{3}\right)$ & $Q_{P}$ & $Q_{S}$ \\
\hline Rock & $3,700-5,800$ & $2,660-3,550$ & 2,650 & $10-200$ & $10-90$ \\
Silicone rubber jacketing & 1,390 & 138 & 1,050 & 6 & 4 \\
increment & $5-100$ & $5-20$ & - & $5-20$ & $5-10$ \\
& & & & Total models: & 6,000 \\
\hline
\end{tabular}

\subsubsection{Choice of Range of Seismic Parameters for Monte Carlo Inversions}

After determining the preferred model for the initial state $\mathbf{m}_{0}$ (Figure 1c), we performed 6,000 simulations of different velocity models and different attenuation models. Since the volume of the rock changed little in the experiment, we did not consider the variation in the rock density caused by the deformation (the density is fixed to be $2,650 \mathrm{~kg} / \mathrm{m}^{3}$ ). We used constant elastic and anelastic parameters of the silicone rubber jacket whereas we systematically modify the parameters of the rock model for the purpose of creating a large database as shown in Table 2. We first simulate the P-wave velocity from 4,700 to 5,800 m/s with a step of $20 \mathrm{~m} / \mathrm{s}$, and from 3,700 to $4,600 \mathrm{~m} / \mathrm{s}$ with a step of $100 \mathrm{~m} / \mathrm{s}$. We ranged $\mathrm{S}$-wave velocity from 2,660 to 3,550 m/s with a step of 10 m/s (cf. Watanabe \& Higuchi, 2015; Zaima \& Katayama, 2018).

Based on the above considerations, in our first step of data processing, for P-wave waveform simulation, we vary the elastic parameters of $V_{P}, Q_{P}$, and the $V_{P} / V_{S}$ while fixing $Q_{S}$ to be constant. During the P-wave analysis, we vary $V_{P} / V_{S}$ from 1.5 to 1.7 with step equals to 0.05 (cf. Zaima \& Katayama, 2018). For the $S$-wave waveform simulation, we change the elastic parameters of $V_{S}, Q_{S}$, and the $V_{P} / V_{S}$ while keeping $Q_{P}$ constant. During data processing, we derive the best models using Equation 3, refining the range of parameters to reach to the best values. We use preferred models during the first numerical simulations to localized our inversions.

\section{Results}

\subsection{Snapshot of the Wavefield}

Figure 4 shows snapshots of the P-wave strain wavefield in our preferred model for $\Delta \sigma=0$ from $2 \mu$ s to 7 $\mu \mathrm{s}$ with a time step of $1 \mu \mathrm{s}$. These snapshots are cross sections of the wavefield at the same height as the source and receiver positions. At $t=2 \mu \mathrm{s}$, the snapshot shows the direct P-wave radiation from the source towards the antipode. No scattering or conversion at the sample surface occurs at this time. However, due to the side-cut surface, the wavefronts have no longer a regular concentric form. The surrounding low-velocity layer outside the sample partially absorbs energy and partially reflects it. This is why the reflection from the sample surface becomes too weak to interference with the direct P waves. Since our model is not a perfect cylindrical model, the two cut corners of the source side immediately provoke reflections. When the direct wave is reflected at the opposite side $(t=4 \mu \mathrm{s})$, the reflected P phase (PP wave) is generated and propagates back to the source side. Since the receiver side has two cut corners as well, two additional reflected waves are generated and interfere with each other between $5 \mu$ s and $6 \mu$ s (Figures $4 \mathrm{~d}$ and $4 \mathrm{e}$ ). However, the external low-velocity silicone layer traps some energy of the direct waves, and the reflections get smaller and smaller. The P-wave propagation snapshot at a later time $(t=7 \mu \mathrm{s})$ shows a complex feature due to the contamination of reflected and converted waves developed at the side-cut surface.

Figure 5 presents the snapshots of S-wave wavefield. Most of side-cut geometry effects mentioned above can also be seen in S-wave snapshots. The $\mathrm{S}$ wave arrives at the receiver at about $6 \mu \mathrm{s}$. What differs the S-wave propagation from that of $\mathrm{P}$ waves is the strong influence from guided waves along the sample surface. The direct $\mathrm{S}$ wave (body wave) at the antipode receiver is contaminated by the waves coming subsequently along the interface (quasi-surface waves) (Figure 5e). This indicates the difficulty of extracting information on the interior of the sample using $\mathrm{S}$ waves alone. 
(a) $t=2 \mu s$

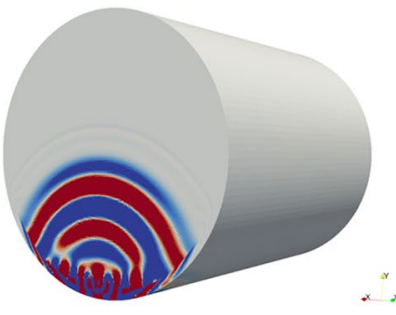

(d) $\mathrm{t}=5 \mu \mathrm{s}$

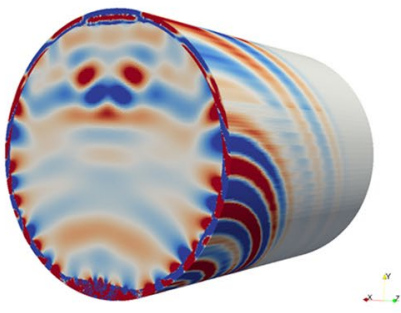

(b) $t=3 \mu s$

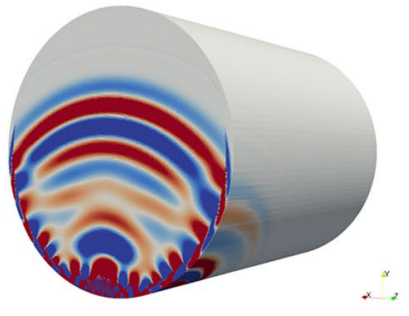

(e) $\mathrm{t}=6 \mu \mathrm{s}$

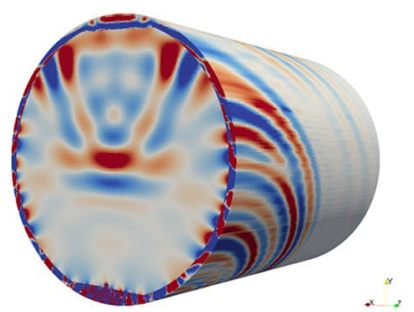

(c) $t=4 \mu s$
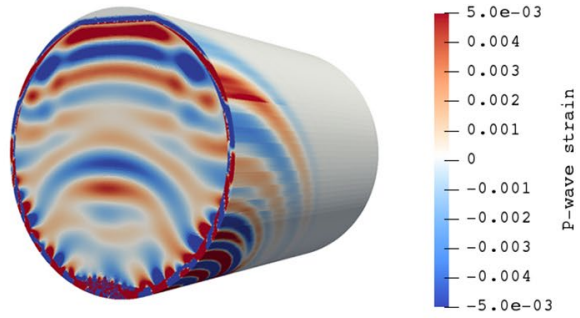

(f) $\mathrm{t}=7 \mu \mathrm{s}$

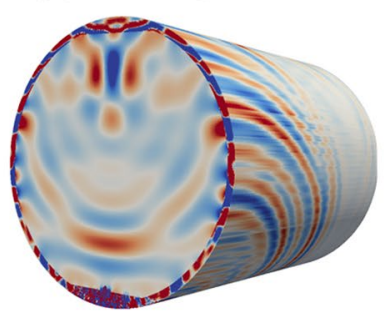

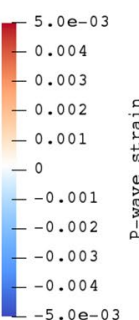

Figure 4. The snapshot of the P-wave wavefield at different time step from (a) 2 to (f) $7 \mu$ s, showing P-wave propagation process inside the medium.

\subsection{Velocity Model Evolution}

Figure 6 shows the waveform fitting results on dry sample using different cost functions: (a) zero-lag cross correlation, (b) $L_{1}$-norm and (c) $L_{2}$-norm. Note that Equation 3 uses reciprocals of objective (b) and (c) when we combine three objective functions together. Regardless of the objective function type, blue color indicates better waveform fits and red color indicates disagreements between observed and synthetics. Among these three cost functions, zero-lag cross correlation can localize best models with less deviation since

(a) $t=2 \mu s$

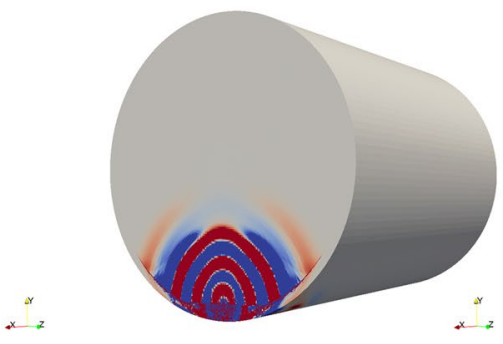

(d) $t=5 \mu s$

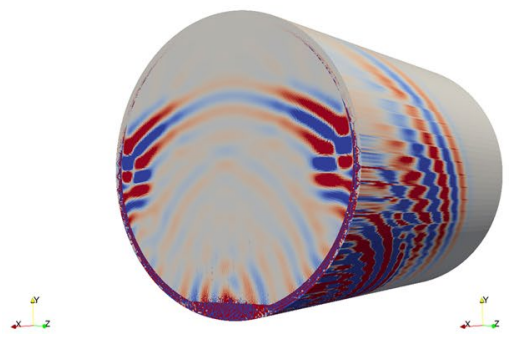

(b) $t=3 \mu s$

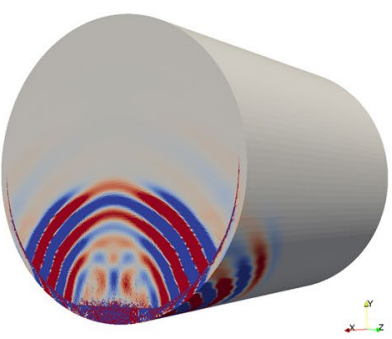

(e) $t=6 \mu s$

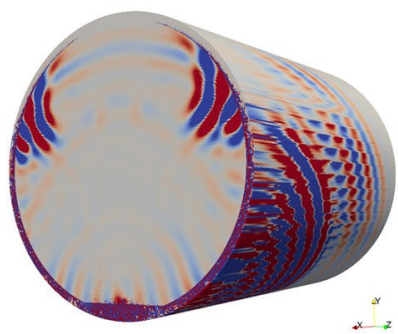

(c) $t=4 \mu s$

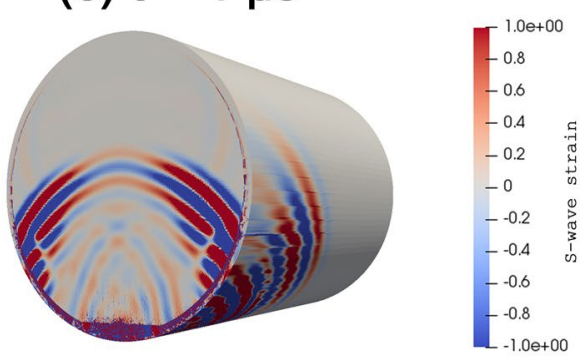

(f) $t=7 \mu s$

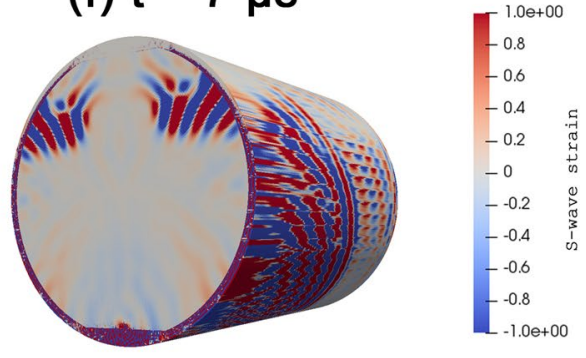

Figure 5. The snapshot of the S-wave wavefield at different time step from (a) 2 to (f) $7 \mu$ s, showing S-wave propagation process inside the medium. 
(a)

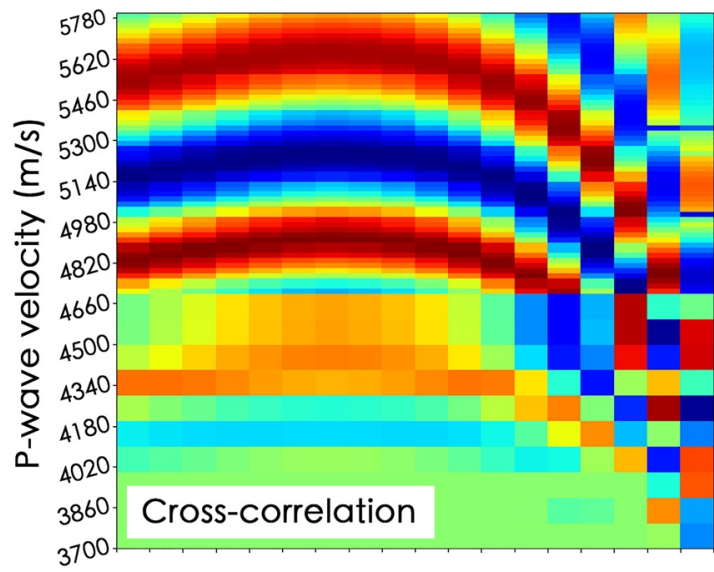

(b)

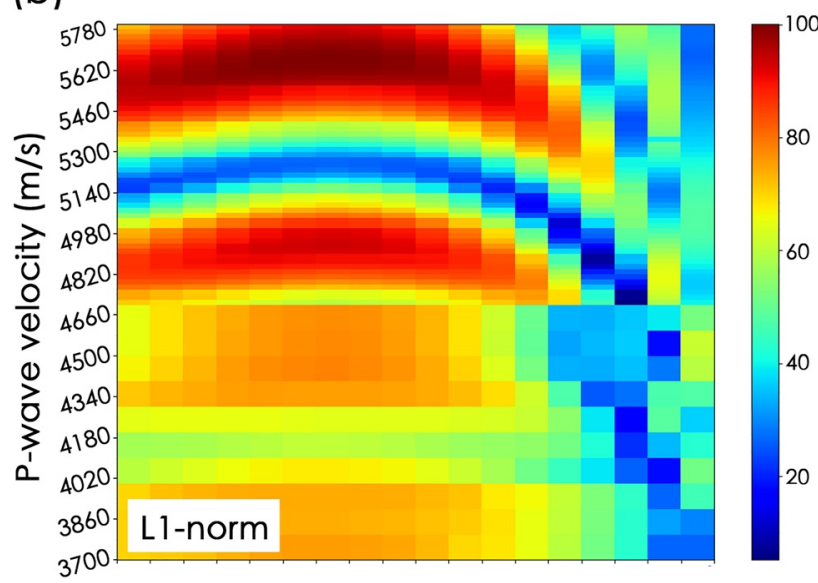

(c)

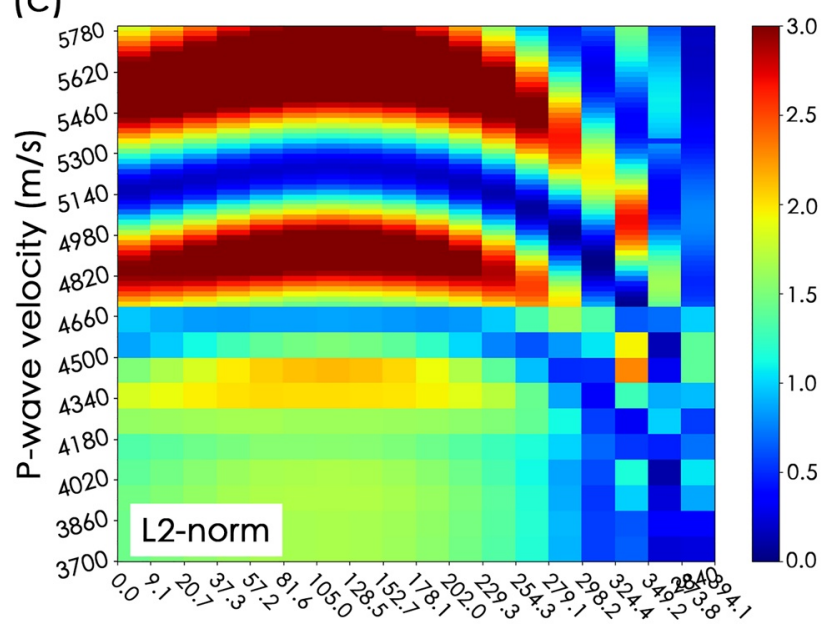

Differential stress (MPa) (d)

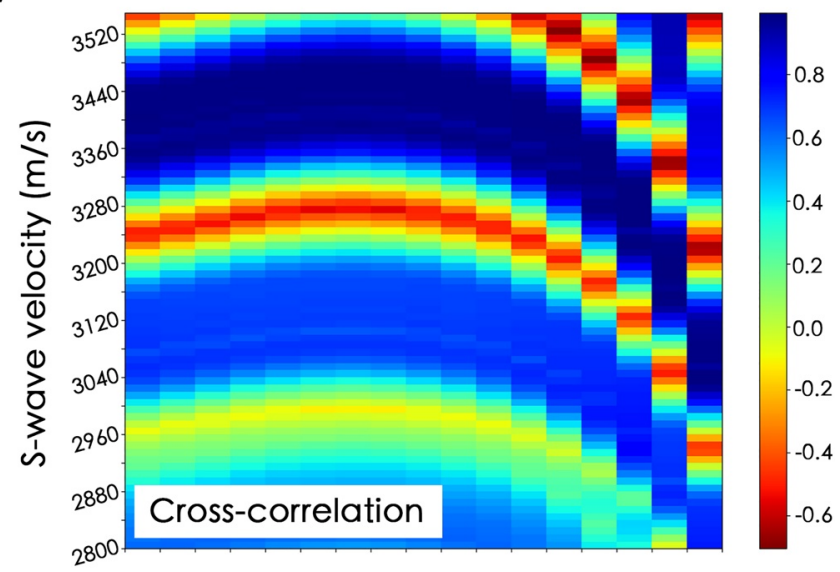

(e)

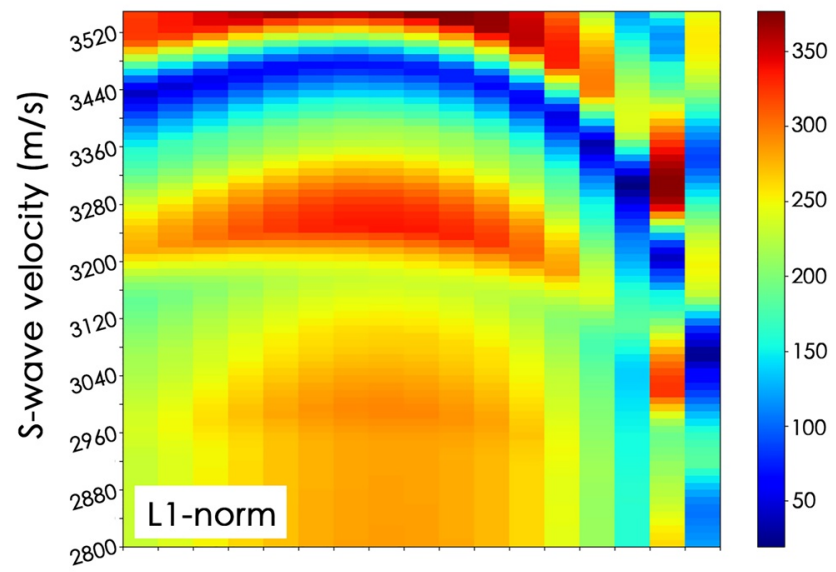

(f)

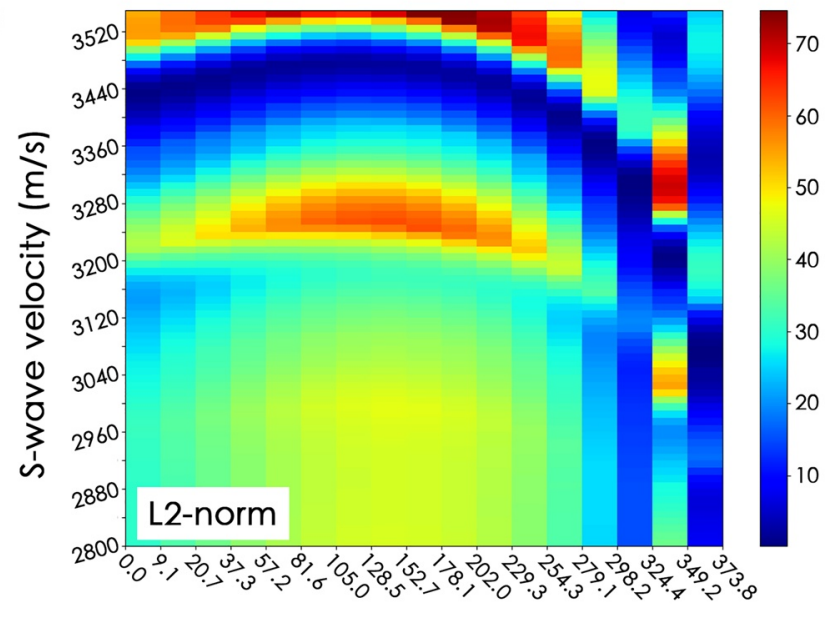

Differential stress (MPa)

Figure 6. The results of waveform fitting using different cost functions for (a-c) P- and (d-f) S-wave, respectively. (a), (d) Zero-lag cross correlation, (b), (e) L1-norm and (c), (f) L2-norm.

kinematic traveltimes are important at the first place (cf. Fuji et al., 2021). $L_{1}$-norm and $L_{2}$-norm both yield a poorer waveform matching at higher compression stress conditions than at lower stress conditions, which indicates the need for further parameter search, such as 3D heterogeneity and/or attenuation. P- and S-wave waveform fitting results on the dry sample are shown in Figure 7. As we discussed in Section 3.1, S-wave 


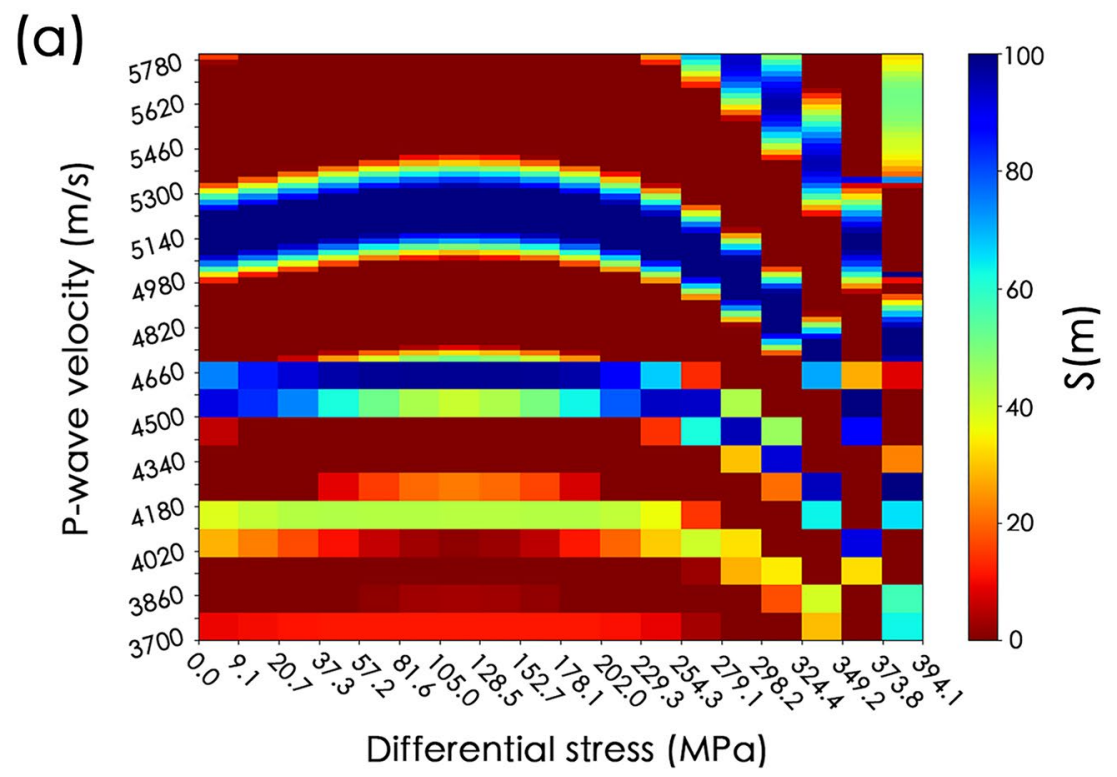

(b)

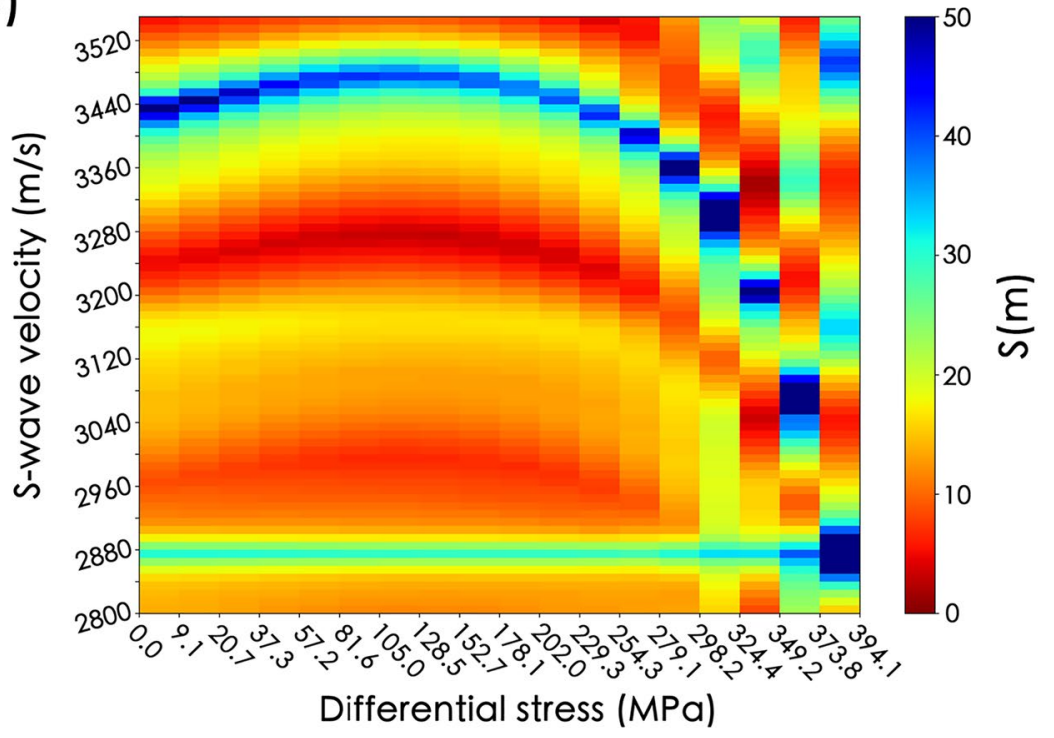

Figure 7. The result of waveform fitting using objective function (Equation 3). The possible trend of change in (a) P-wave and (b) S-wave velocity with increasing differential stress is shown as the high extreme of the scale (dark blue).

waveform is more difficult to fit than P-wave waveform. Figure 8a shows the comparison of the best-fit $V_{P}$ and $V_{S}$ models of the dry sample as well as the hand-picking results from Zaima and Katayama (2018), indicating the evolution of the P- and S-wave velocity. For the experiment under dry conditions, the evolution of $V_{P}$ and $V_{S}$ in general are similar, and follows three stages: (a) increasing stage, where $V\left(=V_{P}, V_{S}\right)$ increases as the $\Delta \sigma$ increases; (b) transition stage, $\Delta V$ remains small; and (c) decreasing stage, $V$ drops rapidly. The highest P-wave velocity structure of dry experiment occurs when the difference between compression stress and confining pressure equals to 81-128 MPa. When P-wave velocity reaches the highest, $\mathrm{P}$ wave remains stable. After $\Delta \sigma=250 \mathrm{MPa}, V_{P}$ drops abruptly, and the sample fails. The peak of $V_{S}$ value occurs at $\Delta \sigma=105 \mathrm{MPa}$ slightly after that of $V_{P}(81 \mathrm{MPa}) . V_{S}$ slowly increases until the differential stress reaches its peak value and decreases until $\Delta \sigma=250 \mathrm{MPa}$, where the $V_{S}$ starts dropping suddenly. In dry experiment, the difference between the fastest and slowest $V_{P}$ is $940 \mathrm{~m} / \mathrm{s}(\sim 18 \%)$. As for $V_{S}$, the difference is about $560 \mathrm{~m} / \mathrm{s}(\sim 16 \%)$. The increase in velocity is larger for compressional waves $(\sim 100 \mathrm{~m} / \mathrm{s})$ than for shear waves $(\sim 40 \mathrm{~m} / \mathrm{s})$ (Figure 8a). 
(a)

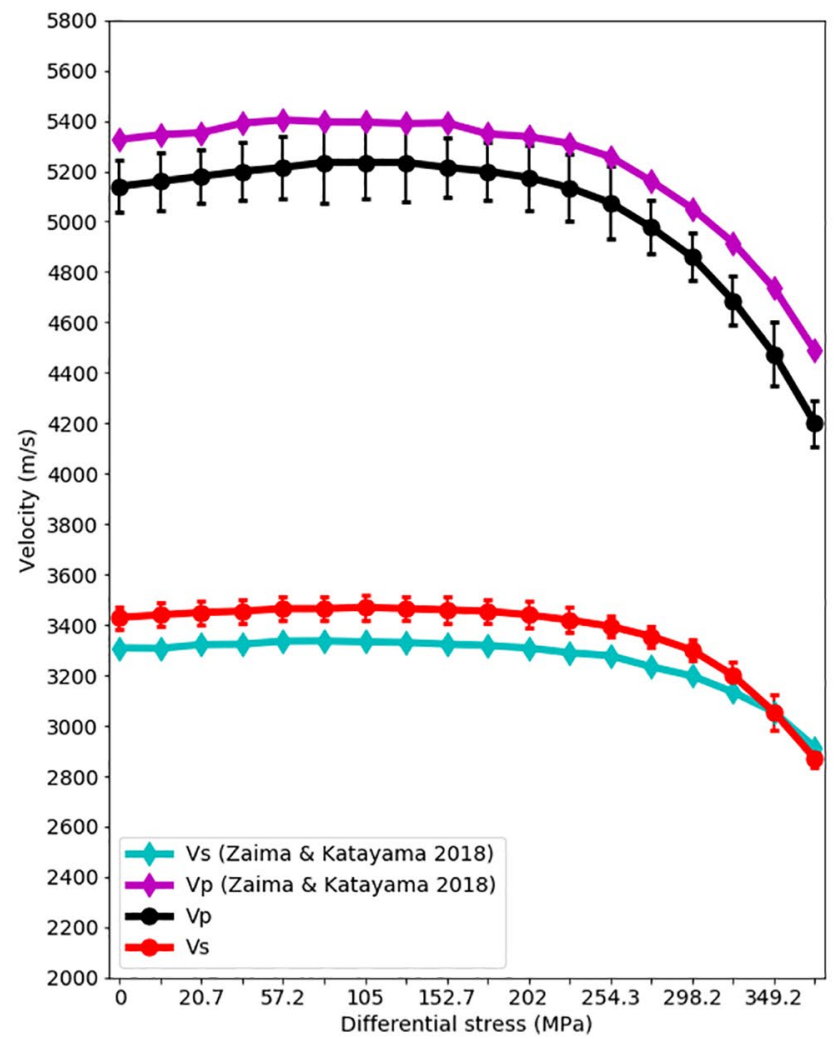

(b)

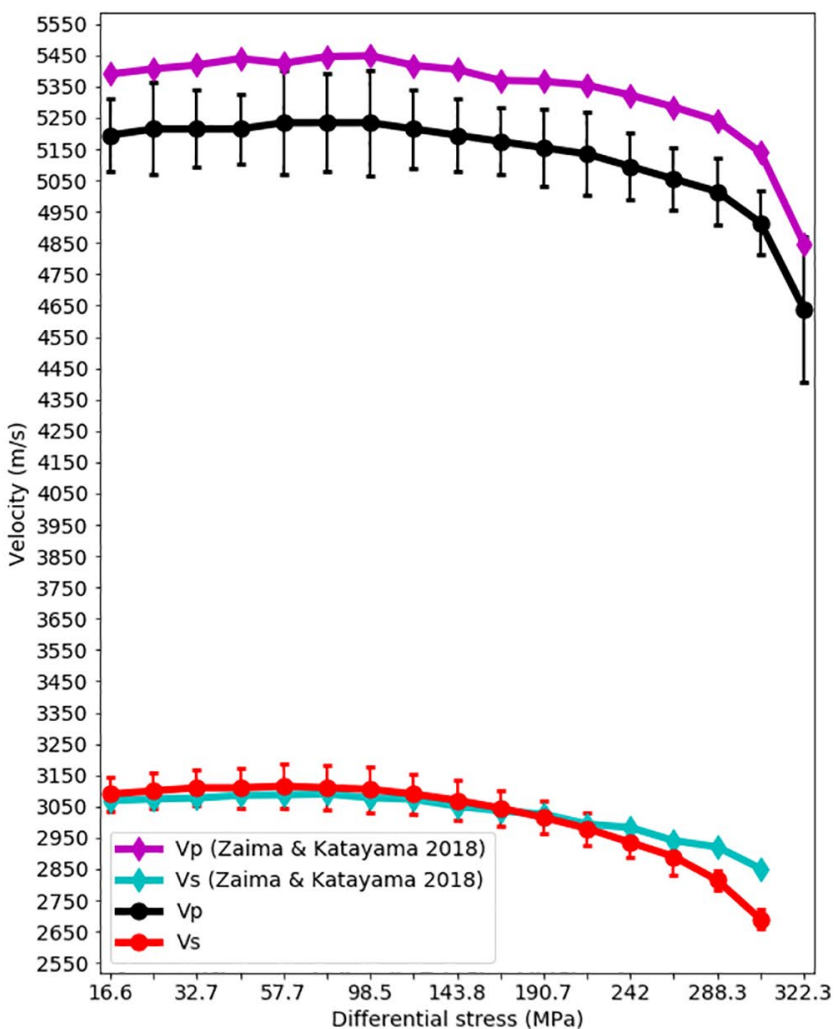

Figure 8. Evolution of $V_{P}$ and $V_{S}$ of (a) dry and (b) wet data. The black and red circle represent the numerical simulated best-fit results of $V_{P}$ and $V_{S}$, respectively. The diamond shapes are the results from Zaima and Katayama (2018), which are calculated from the hand-picked first arrivals.

Similarly, the velocity evolution of the wet sample (Figure $8 \mathrm{~b}$ ) follows the three stages of dry sample. In wet experiment, the difference between the maximum and the minimum $V_{P}$ is $320 \mathrm{~m} / \mathrm{s}(\sim 6.1 \%)$ and the $V_{S}$ difference is about $425 \mathrm{~m} / \mathrm{s}(\sim 13.7 \%)$. Figure 9 shows that increasing compression stress has larger impact on $V_{P}$ changes in dry data than in the wet data. Among all the data sets, the $d V_{S}$ of wet data decreases most when having the same stress stage. Furthermore, the turning point of velocity changes of wet data shows earlier ( $60 \mathrm{MPa})$ than dry data ( 100-150 MPa). The increasing and transition stages of the wet data are much shorter than those of dry data.

\subsection{Attenuation Parameters Evolution}

The variations of $Q_{P}^{-1}$ and $Q_{S}^{-1}$ related to the increasing compression stress are compared in Figure 10a. For both dry and wet experiments, attenuation shows a different behavior compared to velocity during the increase of effective stress. For dry experiment, $Q_{P}$ varies initially from 250 to $10\left(Q_{P}^{-1}\right.$ varies from $4 \times 10^{-3}$ to $\left.1 \times 10^{-1}\right)$ and $Q_{S}$ varies from 100 to $20\left(Q_{S}^{-1}\right.$ varies from $1 \times 10^{-2}$ to $\left.5 \times 10^{-2}\right)$ during the deformation. Unlike velocity change, $Q_{P}^{-1}$ has a minor variation when $\Delta \sigma$ varies from 0 to $298.2 \mathrm{MPa}$. Only after $\Delta \sigma$ reaches $254 \mathrm{MPa}, Q_{P}^{-1}$ starts to rise. When $\Delta \sigma$ exceeds $324 \mathrm{MPa}, Q_{P}^{-1}$ grows rapidly. $Q_{S}^{-1}$ has a similar behavior as $Q_{P}^{-1}$ during rock deformation. Yet, $Q_{S}^{-1}$ rises greatly when $\Delta \sigma$ exceeds $298.2 \mathrm{MPa}$, which is later than the $Q_{P}^{-1}$. It shows that the internal structural changes of the rock have a greater impact on the attenuation of $\mathrm{P}$ waves in dry case. For the wet experiment, $Q_{P}$ varies initially from 130 to 170 , and from 170 to 20 ( $Q_{P}^{-1}$ varies from $7.7 \times 10^{-3}$ to $5.9 \times 10^{-3}$, and from $5.9 \times 10^{-3}$ to $\left.5 \times 10^{-2}\right)$. $Q_{S}$ varies from 80 to 110 , and from 110 to $20\left(Q_{S}^{-1}\right.$ varies from $1.3 \times 10^{-2}$ to $9.1 \times 10^{-3}$, and from $9.1 \times 10^{-3}$ to $5 \times 10^{-2}$ ) along the increase of differential stress. The $Q$ evolution obtained from wet data is particular: the attenuation decreases first and then increases, which could be coherent with the evolution of the velocity. Since, the water-saturated sample breaks earlier than 


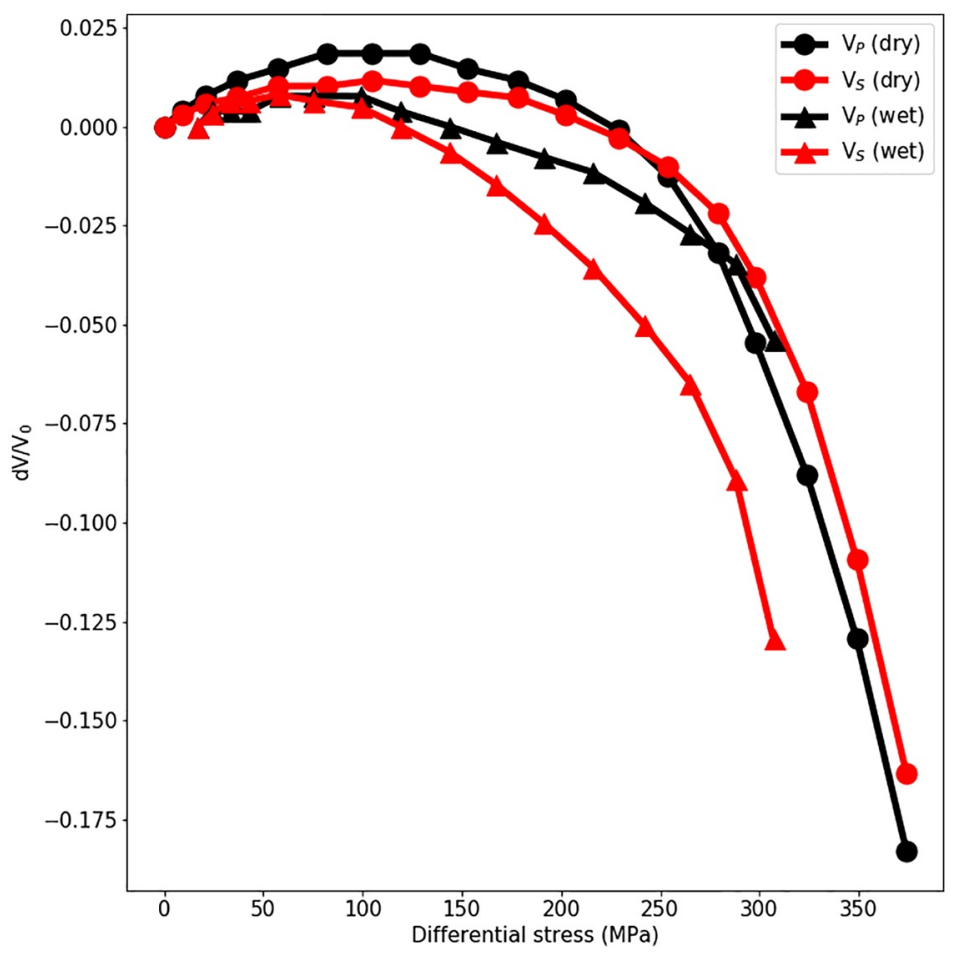

Figure 9. Ratios of differential velocities to initial velocity $\left(d V / V_{0}\right)$, showing the velocity change compared to the original stage at each stress condition.

the dry sample, the wet sample could have experienced less cracks and therefore causes the difference between the $Q$ evolution in dry and wet experiments.

Since, we do not know the absolute amplitude of the wavelet input and the conversion formula of the transducer, we cannot discuss the absolute value of $Q$ and hence we discuss $Q^{-1}$ variation. Figure 10b compares the $Q^{-1}$ variation between different experiments and shows the relative change of $Q^{-1} / Q_{0}^{-1}$. The relative change of $Q^{-1} / Q_{0}^{-1}$ shows that the attenuation of saturated rock is stronger than the dry rock. In dry rock, $Q_{P}<Q_{S}\left(Q_{P}^{-1}>Q_{S}^{-1}\right)$ during the compression. On the contrary, $Q_{P}>Q_{S}\left(Q_{P}^{-1}<Q_{S}^{-1}\right)$ during the compression
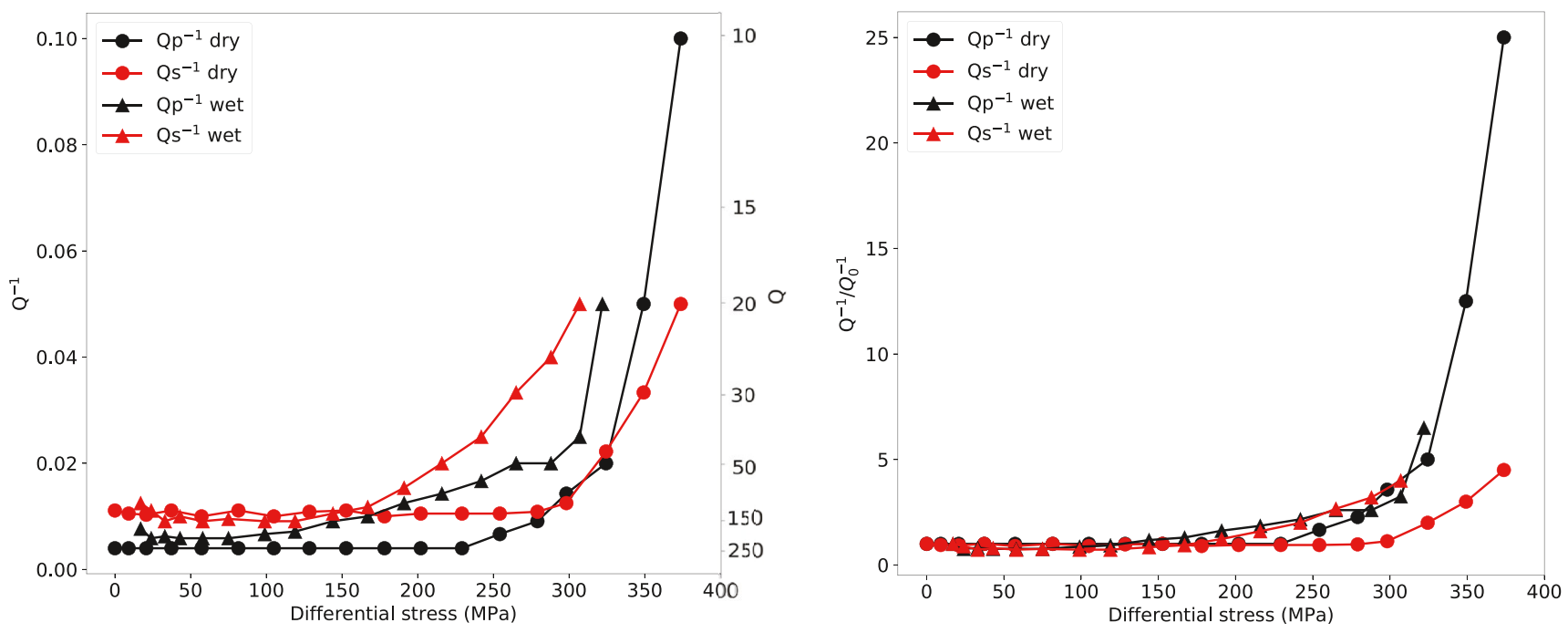

Figure 10. (a) $Q^{-1}$ of wet and dry data, (b) Ratios of differential $Q^{-1}$ to initial $Q^{-1}\left(Q^{-1} / Q_{0}^{-1}\right)$ of wet and dry data. 
(a)

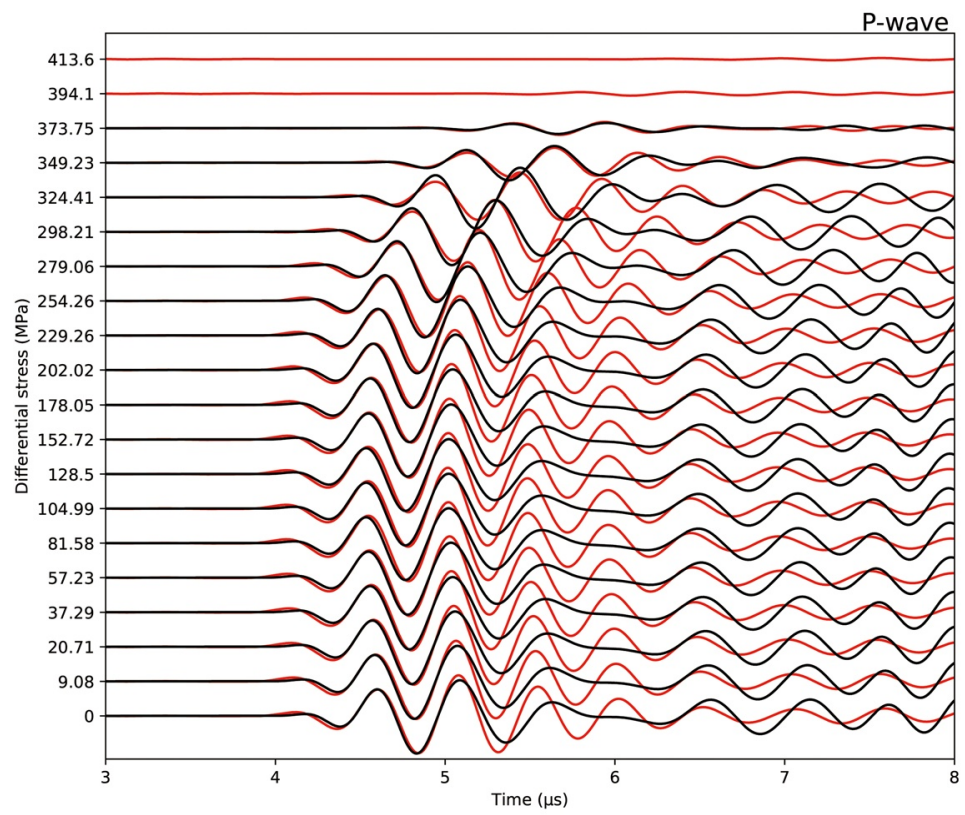

(b)

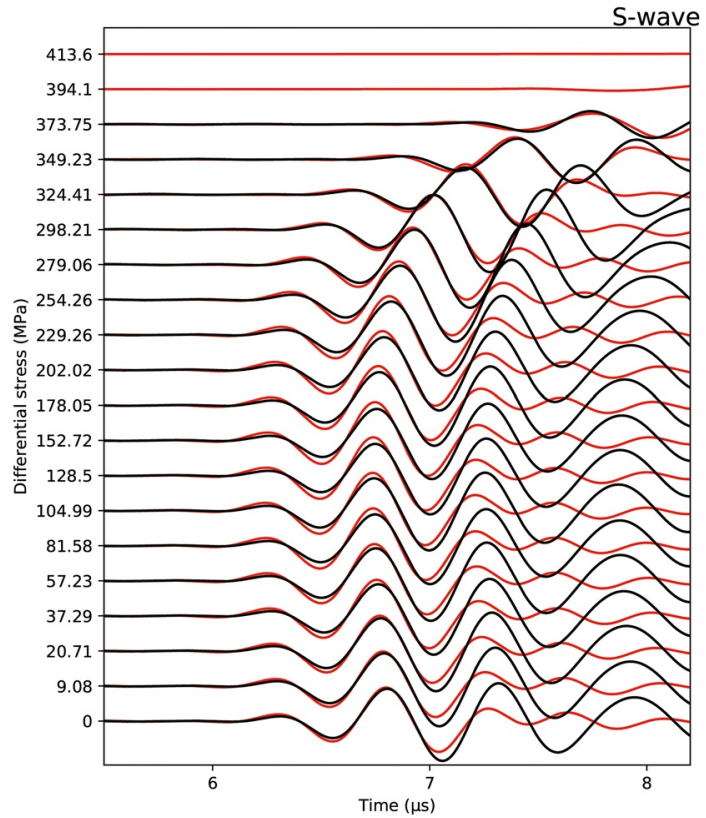

Figure 11. The result of (a) $\mathrm{P}$ - and (b) S-wave strain waveform fitting of dry data using Equation 3 to find the best-match model with proper $V_{P}, V_{S}, Q_{P}, Q_{S}$. The black lines are the best-match synthetic waveforms. The red lines are the experimental data. The $y$-axis indicate the deformation progress and correspond to a condition of $\Delta \sigma$. The $x$-axis represents the time of wave propagation.

in water-saturated rock. Under the same compressional pressure, attenuation of wet sample is higher than dry sample. In general, the wet sample is more sensitive to the attenuation variation.

\subsection{Further Waveform Analysis}

In this subsection, we observe the waveform discrepancies between the synthetics and observed data. The first observation is that the first arrivals of both the P- and S-wave waveforms gradually arrived earlier and later as the compressional pressure increases. In Figures 11a and 12a, the first three peaks of the synthetic P-strain waveforms fit well with the experimental data. The synthetics do not fit well with the observed data after the fifth peak (i.e., $6 \mu$ s of Figures 11a and $6.8 \mu$ s of Figure 12a) due to the incapability of accurate shear-wave modeling. When $\Delta \sigma$ is $349.23 \mathrm{MPa}$ in the case of the dry data set, the previously disappearing fifth peak reappeared in Figures 11a, which may be again due to the poorly constrained shear waves. In laboratory, the $\mathrm{S}$ waves seem to have less effects on the recorded P-wave strain waveforms. When $\Delta \sigma$ exceeds 373.75 MPa in the case of the dry data, the interior structure of the rock is discontinuous and, hence, difficult to homogenize, what makes our modeling inefficient. In Figures $11 \mathrm{~b}$ and $12 \mathrm{~b}$, synthetic S-strain waveforms match well with the first two peaks experimental waveforms. After the third peak, the experimental waveforms have more peaks and smaller amplitudes, causing the synthetic waveforms to fail to match the experimental data. However, the matching of the first few peaks provided enough information on the velocity model and attenuation parameters change.

\section{Discussion}

In this study, we developed a methodology to invert time-lapse active seismic data in laboratory in order to infer the changes of the elastic and anelastic parameters. Here, we first discuss the numerical configuration to yield synthetics sufficiently comparable against the observed data. Thence, we resume the observation and make further interpretation on our experiments and show the need for 3D waveform inversions in near future. 
(a)

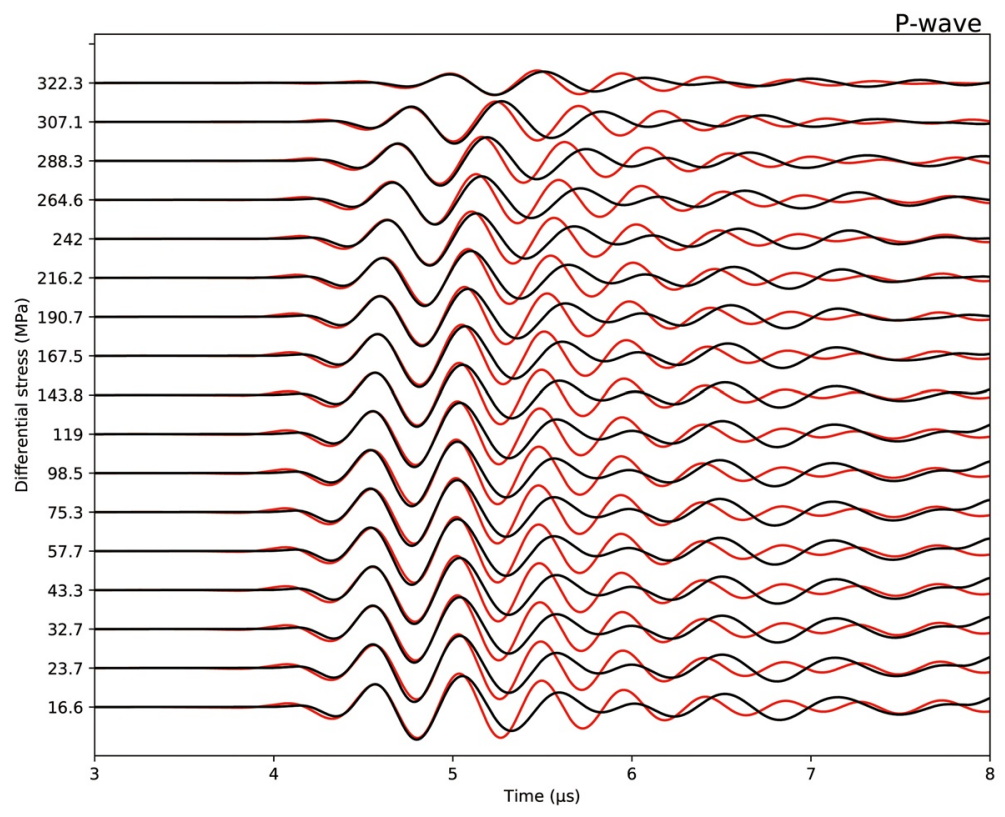

(b)

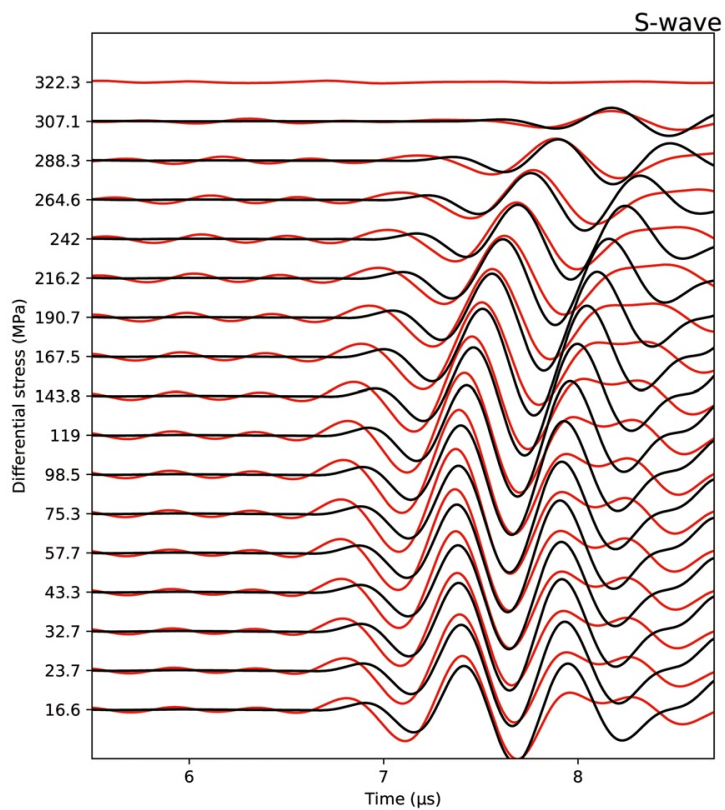

Figure 12. The result of (a) P- and (b) S-wave strain waveform fitting of wet data using Equation 3 to find the best-match model with proper $V_{P}, V_{S}, Q_{P}, Q_{S}$. The black lines are the best-match synthetic waveforms. The red lines are the experimental data. The $y$-axis indicate the deformation progress and correspond to a condition of $\Delta \sigma$. The $x$-axis represents the time of wave propagation.

\subsection{Effect of the 3D Model Settings and Geometry}

There have been few studies that simulated elastic wave propagation at centimeter scales and compared the synthetics against observed data. Yoshimitsu et al. (2016) is one of the few examples and they performed their experiments on a stationary aluminum sample while we did for deforming granites. In addition, they used lasers for both a source and a receiver while we had to use transducers due to the deformation experiments configuration. They observed the body- and surface-wave propagation being influenced by the cylindrical geometry. In our numerical modeling with free surface conditions, we could observe the same phenomenon of large-amplitude quasi-surface waves emerged along the curved surface of the sample after the body waves. However, in our experimental data, these large-amplitude surface waves were not present due to the surrounding glue layer. In order to reproduce their absence, we had to introduce special boundary conditions. While Yoshimitsu et al. (2016) added absorbing boundary conditions on the top and at the bottom, we introduced an additional layer with physical properties of silicone surrounding the target rock model, making waveforms much more realistic (Figure 1c). Furthermore, we found that the two cut sides parallel to compressional pressure are an indispensable condition to take into account. A model with these cut sides provoke an important amount of extra reflections compared to a simple cylindrical model. To conclude, it is important to consider the three-dimensional geometry.

The surrounding silicone rubber layer can provide a buffer-like region, which can slow down and reduce the amplitude of quasi-surface-wave propagation between the two media. Note that when the direct waves reach the interface between the two materials and travel through a low- $Q$ and low-velocity material, the resulting wavefield is amplified. The transmission coefficient calculated to confirm the observed phenomenon is shown in Appendix A.

\subsection{Observed Changes in Wave Velocity and Attenuation}

The evolution of homogenized velocity and attenuation in laboratory under a physical state should provide information on micro structural changes. The simultaneous Monte Carlo inversion for four homogenized elastic and viscoelastic parameters, $V_{P}, V_{S}, Q_{P}$ and $Q_{S}$, were able to eliminate the cross-talks of these 
parameters. While Aji granite comprises an average grain size of $\sim 0.3 \mathrm{~mm}$ (Kudo et al., 1992), the effective wavelength can be estimated as around 1.5-3.0 mm using the apparent dominant frequency ( 1-2 $\mathrm{MHz})$ and shear-wave velocity $(\sim 3,000 \mathrm{~m} / \mathrm{s})$. The local heterogeneity of grain size can therefore be neglected for the first place. However, we would still like to refine the structure using our waveform data but this simple estimation justifies the homogeneous modeling in this preliminary study.

The variation of wave velocity (Figure 8) during deformation estimated from our waveform matching methodology has a similar trend as seen by Zaima and Katayama (2018). Hereafter, we call the $V_{P}$ and $V_{S}$ measured by hand-picking "apparent" $V_{P}$ and $V_{S}$. Our results show slightly lower P-wave and higher S-wave velocities compared to the apparent velocities. However, apparent velocity models and the models obtained by our waveform-matching scheme do not correlate at large stress stage, especially for S-wave velocity. This indicates that the hand-picking fails even for first arrivals due to the ambiguity of travel times for highly dispersed waveforms, whereas waveform matching can still detect the phase arrivals objectively. Figure 9 shows the differential $V_{P}$ and $V_{S}$ obtained using waveform matching with respect to the initial status for dry and wet experiments. Since the dry sample resists to the external stress more than the wet sample, we can observe that the dry sample's velocities drop down to $\sim 15-18 \%$ while the wet sample shows $-5 \%$ for $V_{P}$ and $-12.5 \%$ for $V_{S}$.

Another advantage of this Monte Carlo waveform inversion for elastic and anelastic parameters is the direct measurements of $Q_{P}$ and $Q_{S}$. While Toksöz et al. (1979) studied the spectral ratios of waveform records of rock experiment relative to the waveform of reference sample, Lockner et al. (1977) and Zaima and Katayama (2018) measured the amplitude ratios of the first and second peaks in order to indirectly infer the attenuation, failing to obtain quality factors. Including $Q_{P}$ and $Q_{S}$ in the simultaneous simulations, objective functions show local maxima, especially for $L_{1}$-norm and $L_{2}$-norm. In particular, synthetic waveforms ignoring $Q_{P}$ and $Q_{S}$ are not comparable to the highly compressed experimental data. The reason of these differences may be due to seismic dispersion induced by attenuation in viscoelastic media, which certainly have more impact on shear waves than on compressional waves. Kjartansson (1979) modeled the relationship between velocity and the quality factor and obtained the increases of the velocity with frequency and $Q^{-1}$. When looking at the discrepancy between S-wave velocities at the last stage of both dry and wet experiments obtained with hand-picking and waveform matching including attenuation, "apparent" values are constantly higher than our results. This is because of the waveform broadening due to low $Q$ at the last stage.

Since Zaima and Katayama (2018) measured only the amplitude ratios of first and second peaks to infer the attenuation, we cannot directly compare our results to theirs. Nevertheless, the amplitude of the S-wave is reduced more than that of the $\mathrm{P}$-wave during deformation under saturated wet conditions, which is consistent with our results (Figure 10). Both P- and S-wave quality factor evolutions of wet data show roughly the same trend as the velocity evolution. The velocity increases while $Q$ increases, and $Q$ grows while the velocity grows.

\subsection{Rock Physical Interpretation}

The simplest way of interpreting the general trend of velocity changes is presented in many studies. At low confining pressure, the micro cracks in the experimental sample can be regarded as randomly distributed. The second stage is the closing process of horizontal micro cracks, which are perpendicular to the maximum stress, leading to velocity increase. The final stage, the vertical cracks form before the failure. However, as the closure of horizontal micro-cracks has little effect on the energy dissipation of $\mathrm{P}$ and $\mathrm{S}$ waves, it is difficult to see the difference during the attenuation process.

Experimental studies found seismic wave attenuation in dry rocks was sensitive to intrinsic material anelasticity, thermoelastic effects, friction at grain boundaries and micro-cracks development (e.g., Gordon \& Davis, 1968; Guéguen \& Schubnel, 2003; Johnston et al., 1979; Simmons \& Brace, 1965; Tisato \& Quintal, 2014; Walsh, 1965; Winkler \& Nur, 1982). Water-saturated rocks, on the other hand, could accommodate fluid-related mechanism that would provoke energy dissipation, which should be responsible for seismic attenuation. Winkler and Nur (1982) declared that the effect of frictional sliding to the attenuation is negligible for pore-fluid rocks. Hence, they emphasized the importance of the role of intercrack fluid flow, which 
may be considered as the major cause of the attenuation. In their experiments of dry, partially saturated, and saturated rock, they showed that S-wave attenuation increases with the degree of saturation and reaches its maximum at total saturation. However, as saturation continues to increase to more than $90 \%$ water saturation, $Q_{P}$ is higher than during partial saturation condition and $Q_{P}>Q_{S}$. These observations can be explained with local fluid flow mechanisms. Guéguen and Schubnel (2003) also referred to the importance of local fluid flow and squirt flow to the attenuation and anisotropy. They numerically obtained dispersion for two different transversely isotropic distribution of cracks. When cracks are aligned vertically, and therefore orthogonally to the source-receiver horizontal plane, SH waves are more dispersive than P waves.

Figure 9 compares the velocity evolution of dry and fully saturated wet experiments. The velocity results show that the presence of saturated water accelerates the rock sample to reach its maximum velocity, which we think is the non-crack mode. The presence of water also causes the fast reduction of shear waves. Figure 10 shows the waveform fitting results of dry and wet experiments, showing that attenuation changes with the increasing compression stress under different water conditions. The wet $\mathrm{S}$ wave shows a greater decrease in both velocity and quality factor, compared with the $\mathrm{P}$ wave, from low pressure until the rupture. These changes in the elastic and anelastic behavior are consistent with the observation of Winkler and Nur (1982), who speculated these effects as intercracks flow. At the beginning, increasing of pressure causes little effect on both the P- and S-wave attenuation of dry and wet samples, because there is no enough density of cracks that affect the wave propagation. As the effective stress increases, our results show that $Q_{P}>Q_{S}$ in the wet case, whereas $Q_{P}<Q_{S}$ for the dry case. This may be produced when there is a compressional pressure high enough to form micro-cracks with higher density, especially with micro-cracks aligned vertically along the long axis. These highly dispersed waves lead to a large amount of attenuation. The characteristics and the local pore fluid flow can explain our attenuation results obtained from fully saturated sample (Guéguen \& Schubnel, 2003; Winkler \& Nur, 1982).

The contribution to attenuation in the dry rock is assumed to be only due to the friction and the intrinsic aggregate attenuation. Intrinsic attenuation is thought to be caused by energy dissipation due to friction at cracks where those faces are barely in contact, making amplitude changes sensitive to crack geometry in the specimen (Lockner et al., 1977; Walsh, 1966). Besides, Bonner (1974) presented an increase of shear wave anisotropy in the dry granite caused by cracks oriented parallel or oblique to the compressional stress. The cracks oriented parallel and subparallel to the axis of maximum compression stress have influence on attenuation. However, we can see that many laboratory experiments confirmed that $Q_{P}<Q_{S}$ exists in the dried samples (Johnston et al., 1979). No specific mechanism has been proposed to explain the difference between P- and S-wave attenuation in dry rock.

Figure 13 compares the exact and relative $V_{P} / V_{S}$ ratio obtained from waveform matching with the $V_{P} / V_{S}$ ratio of Zaima and Katayama (2018). A number of studies have mentioned that apparent $V_{P} / V_{S}$ ratio rise can be found in the seismic data of the subduction zone or laboratory experimental data, which may be linked to the high pore fluid pressure and crack anisotropy when approaching failure (Audet et al., 2009; Christensen, 1984; Peacock et al., 2011; Wang et al., 2012). It is worth noting that there is a difference between the velocity variation curve we corrected through attenuation and the apparent $V_{P} / V_{S}$ ratio (Figure 13b). Our results present higher $V_{P} / V_{S}$ ratios for wet experiment when rock approaches rock failure. Even in dry rock experiment, $V_{P} / V_{S}$ ratios are slightly higher compared to the apparent $V_{P} / V_{S}$ ratios before rupture. These phenomena may be caused by the sudden rise of S-wave anisotropy (Bonner, 1974) and thus influencing the attenuation and wave velocity. This characteristic of $V_{P} / V_{S}$ ratio rising marks the particularity of the rock right before failure, and may provide robust information on fracture prediction.

\subsection{Toward 3D Waveform Inversion}

Our method can process large amounts of experimental data quickly and provide the best matching values of $V_{P}, V_{S}, Q_{P}$, and $Q_{S}$. However, the synthetic seismograms cannot match the later phases regardless of parameter sets. For example, the fifth peak $(\sim 6 \mu \mathrm{s})$ of the synthetic waveforms in Figure 11a cannot match the data, especially when it coincides with the arrival time of $\mathrm{S}$ waves. The difficulty may come from model homogenization that should not be valid for scattered S waves. The second possibility is that our boundary condition is still deficient, causing the S-wave waveforms to have poor matching. Since S waves and 
(a)

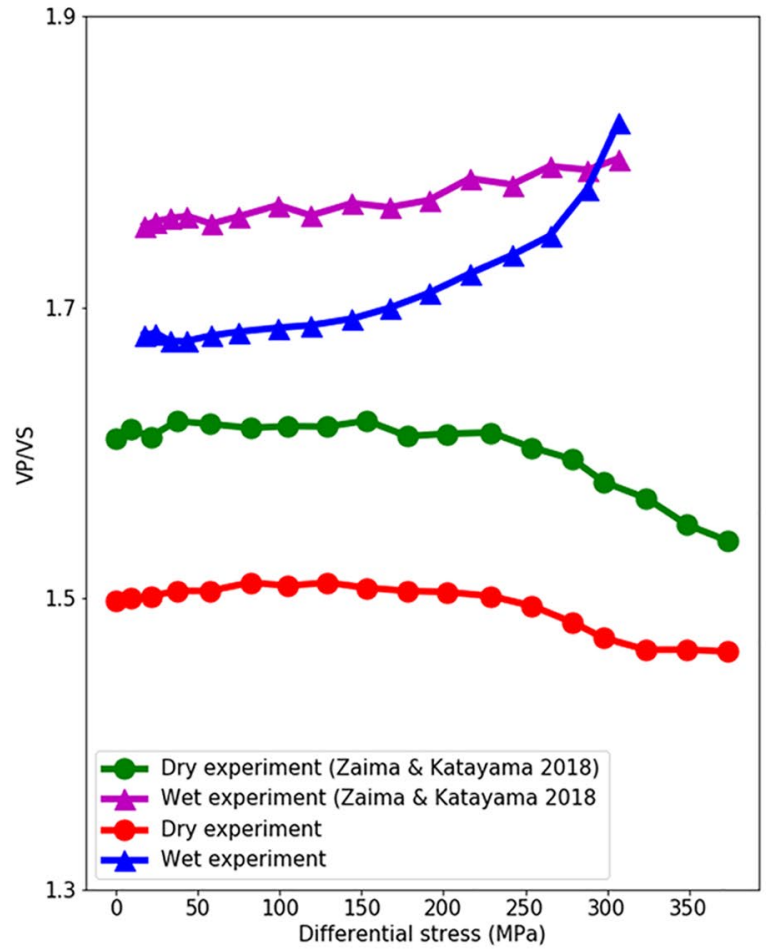

(b)

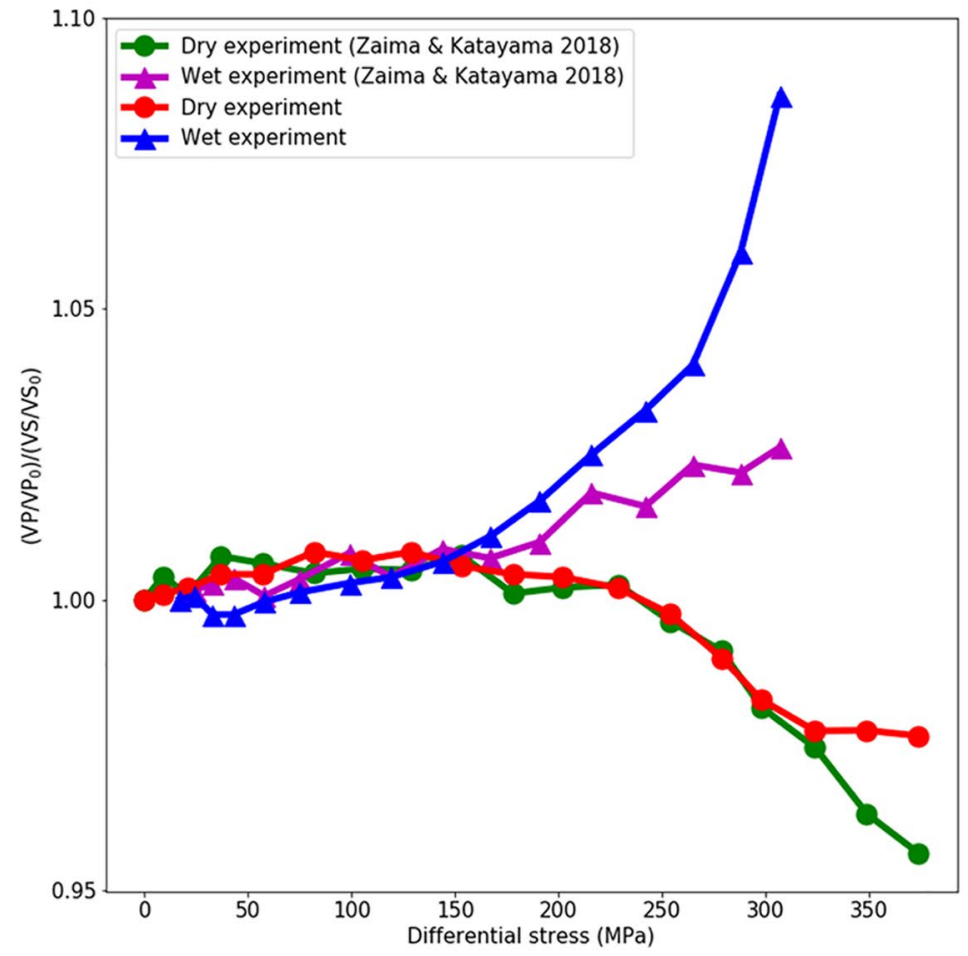

Figure 13. (a) $V_{P} / V_{S}$ ratio change, (b) Relative $V_{P} / V_{S}$ ratio change.

quasi-surface waves are sensitive to smaller heterogeneity than $\mathrm{P}$ waves, it is natural to have this poorer fit in S-wave waveforms.

Therefore, full waveform inverse problem must inevitably move towards the development of heterogeneous models. Our method is an isotropic approximation of the deformed rocks, such that so far we can only match the first few peaks in the waveform to get the elastic and viscoelastic parameters. Kovalyshen et al. (2018); Kovalyshen et al. (2020) estimated anisotropy by inverting elastic wave arrival times for characterizing the damage and failure processes in rocks. They also discussed the effective interpretation and correction of waveforms recorded by the finite-size transducers in laboratory experiments. When we start to extract more information on the weakening of rock materials from the waveform, we must use a three-dimensional heterogeneous model. Indeed, it is only through heterogeneous model that we could better simulate the structural changes and mechanisms inside the rock during deformation.

\section{Conclusions}

Through the matching of the simulated and observed data, we obtained the velocity and attenuation changes as the pressure rises up to rupture in laboratory. Unlike previous studies, the estimation of attenuation no longer requires amplitude analysis but can be directly conducted through our simultaneous inversion schemes, without trade-offs between the unknowns. The "apparent" velocity change could be biased by ignorance of attenuation and therefore our estimation of $V_{P}$ and $V_{S}$ is more trustable. Combining seismology and rock physics, the data measured can explain the fracture nucleation during varying stress conditions. Seismology can bring useful information on small-scale rock fractures. Variations of elastic and anelastic parameters revealed in this study show different trends from the usual rock measurements, these observation and analysis may thus give us the opportunity to understand the rock failure mechanism of not only laboratory experiments, but also the shallower part of the Earth's crust. 


\section{Appendix A: Boundary Condition Tests}

As shown in Figure A1a and A1b, the free-surface boundary rock model cannot reproduce the observations, due to its complete reflections from boundaries. Hence, we attempt to modify the boundary conditions by considering the silicone rubber, as in the real experiments: a layer of glue and silicone rubber is attached to the rock sample on its cylindrical side to jacket the sample. This layer is used to isolate the oil from the rock sample and transducers. The physical parameters of this layer are calculated from the characteristics of silicone rubber used in the experiment, Shin-Etsu KE45 W RTV silicone rubber (Table 1). The layer is added both to the cylindrical and to the side-cut cylindrical rock model. Figure A1 shows the waveforms for (a) the cylindrical rock model with free-surface boundary, without surface cut; (b) a cylindrical model with free-surface boundary, with surface cut; (c) a cylindrical model with silicone layer without surface cut; and (d) a cylindrical model with silicone layer with surface cut. The additional low-velocity layer surrounding the cylindrical rock sample behaves as an ad-hoc modified boundary conditions to absorb some wave energy, while preserving some reflections. Between models (a) and (b) in Figure A1, we can observe the subtle differences caused by the cut sides. However, both of the two models (a) and (b) cannot reproduce the observed waveforms reasonably. Models (c) and (d) have similarity until $5.5 \mu \mathrm{s}$ (the third peak), after which the reflections from the antipodal point is larger than the real data with model (c). Due to the additional reflections back to the inner rock model in (d), the later phases show faster amplitude decay and the synthetics for (d) resemble better to the observed datum than (c). The comparison among the four models demonstrates that the more features added, the more similar the synthetics to the observed waveform. We thus concluded to use model (d) as our boundary conditions.

The additional layer in model (d) with the characteristics of silicone rubber can provide a buffer-like region that slows down the waves and reduce the amplitude of quasi-surface waves propagating between the two different materials. Figure A2 shows the coefficients of $\mathrm{P}$ to $\mathrm{P}$ and $\mathrm{S}$ to $\mathrm{S}$ transmission (Lay \& Wallace, 1995), calculated as amplitude and phase at the solid-solid interface from rock to the silicone rubber. When the

(a)

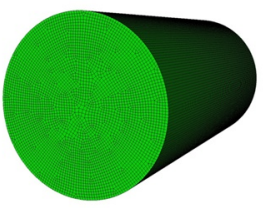

(b)

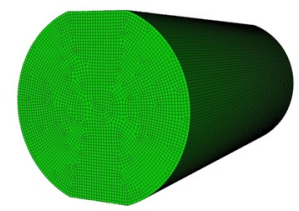

(c)

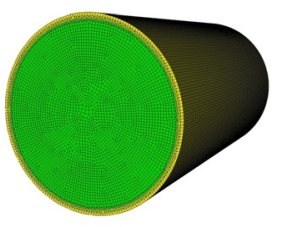

(d)

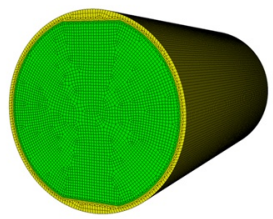

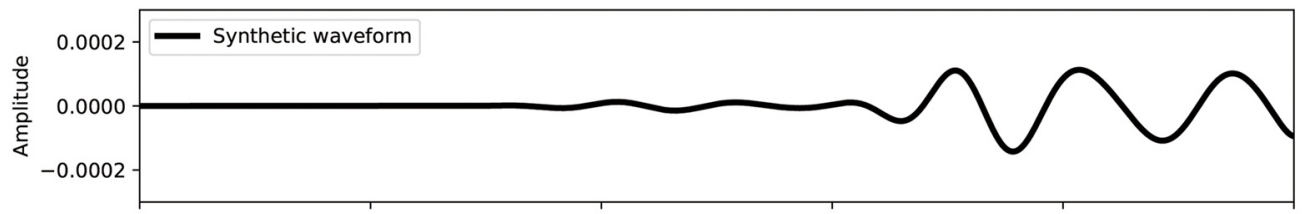
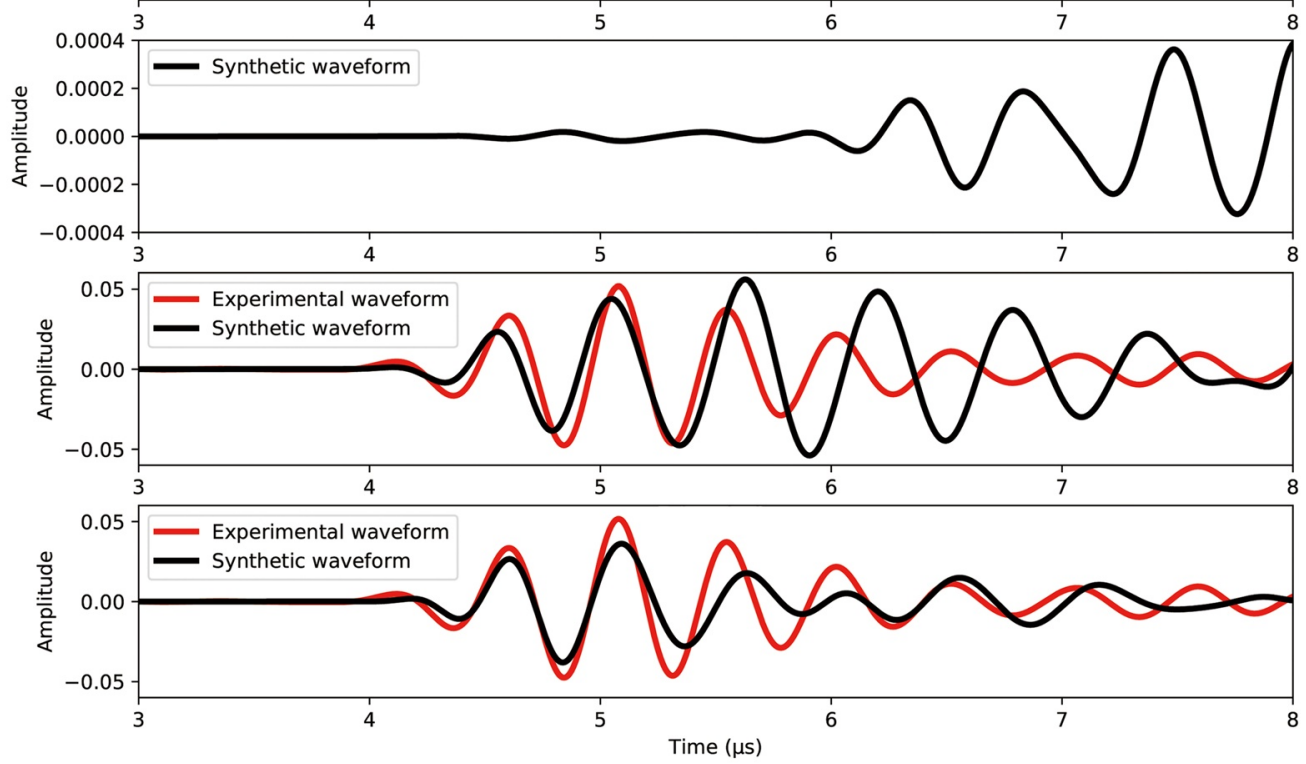

Figure A1. The four models tested in this study and their synthetic waveform (a) cylindrical model with free-boundary, (b) side-cut cylindrical model with free-boundary, (c) cylindrical model with surrounding silicone jacketing model and (d) side-cut cylindrical model with surrounding silicone jacketing model. 
(a)

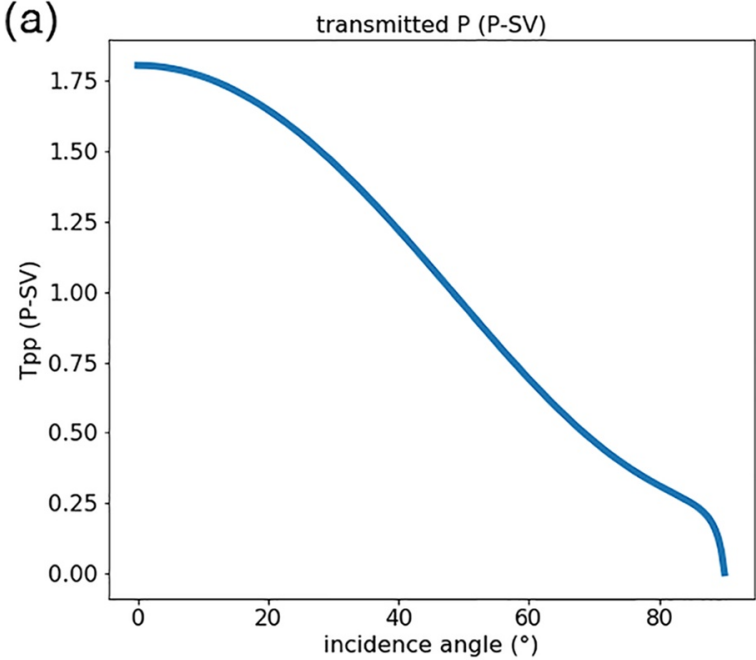

(c)

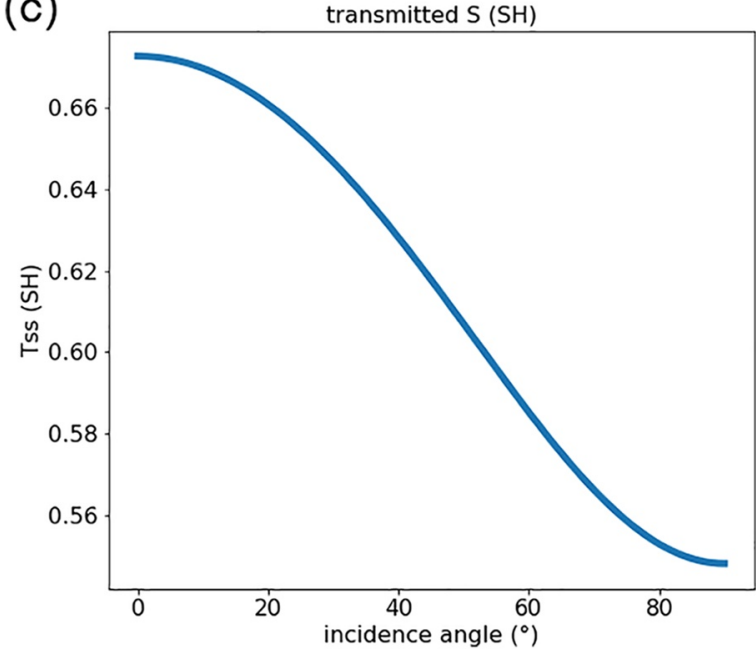

(b)

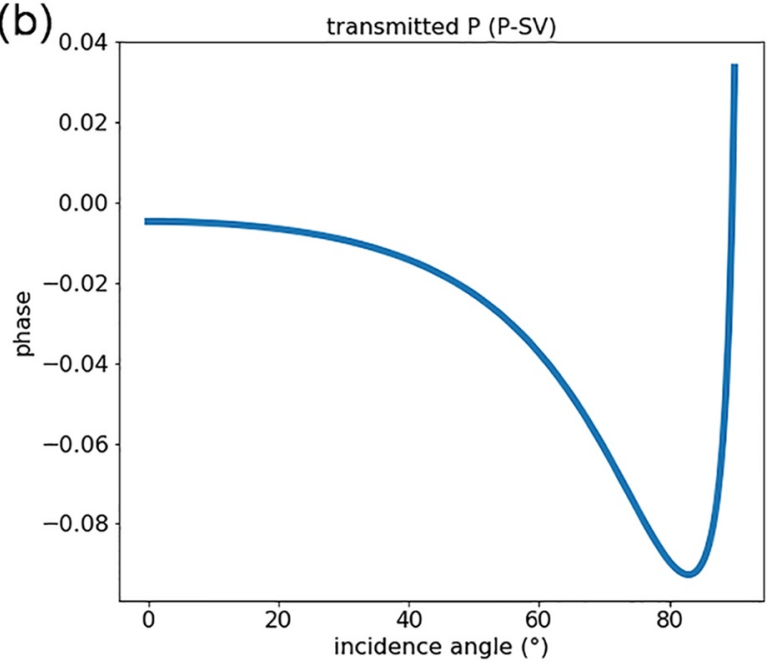

(d)

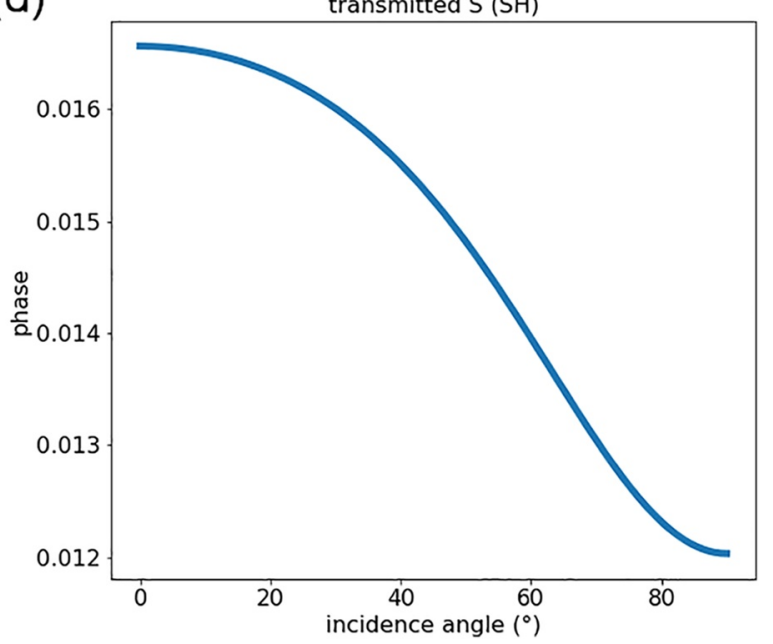

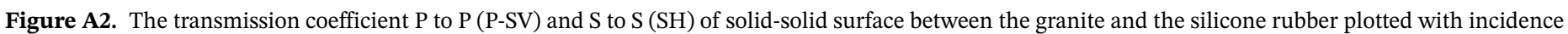

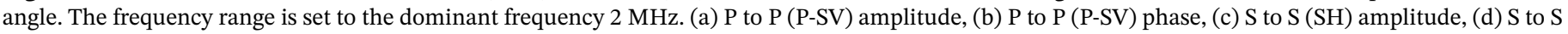
(SH) phase.

incidence angle equals to $0, \mathrm{P}$ to $\mathrm{P}$ transmission coefficient is as high as 1.75 , which is coherent to our observation that the direct $\mathrm{P}$ waves are amplified when they transmit and reach the interface between the two materials and travel through a low- $Q$ and low-velocity material.

\section{Appendix B: Source Wavelet Input}

The source time function is formally taken from the laboratory experiment input waveform. However, the absolute amplitude of the input signal transmitted to the rock by the piezoelectric transducers is unknown. Therefore, in this study, we estimated the effective source time function by a trial-and-error approach. We concluded that the recorded input waveform low-pass filtered at $2 \mathrm{MHz}$ is the best source time function to be used. This is the same dominant frequency band used in laboratory experiment (Figure B1). 

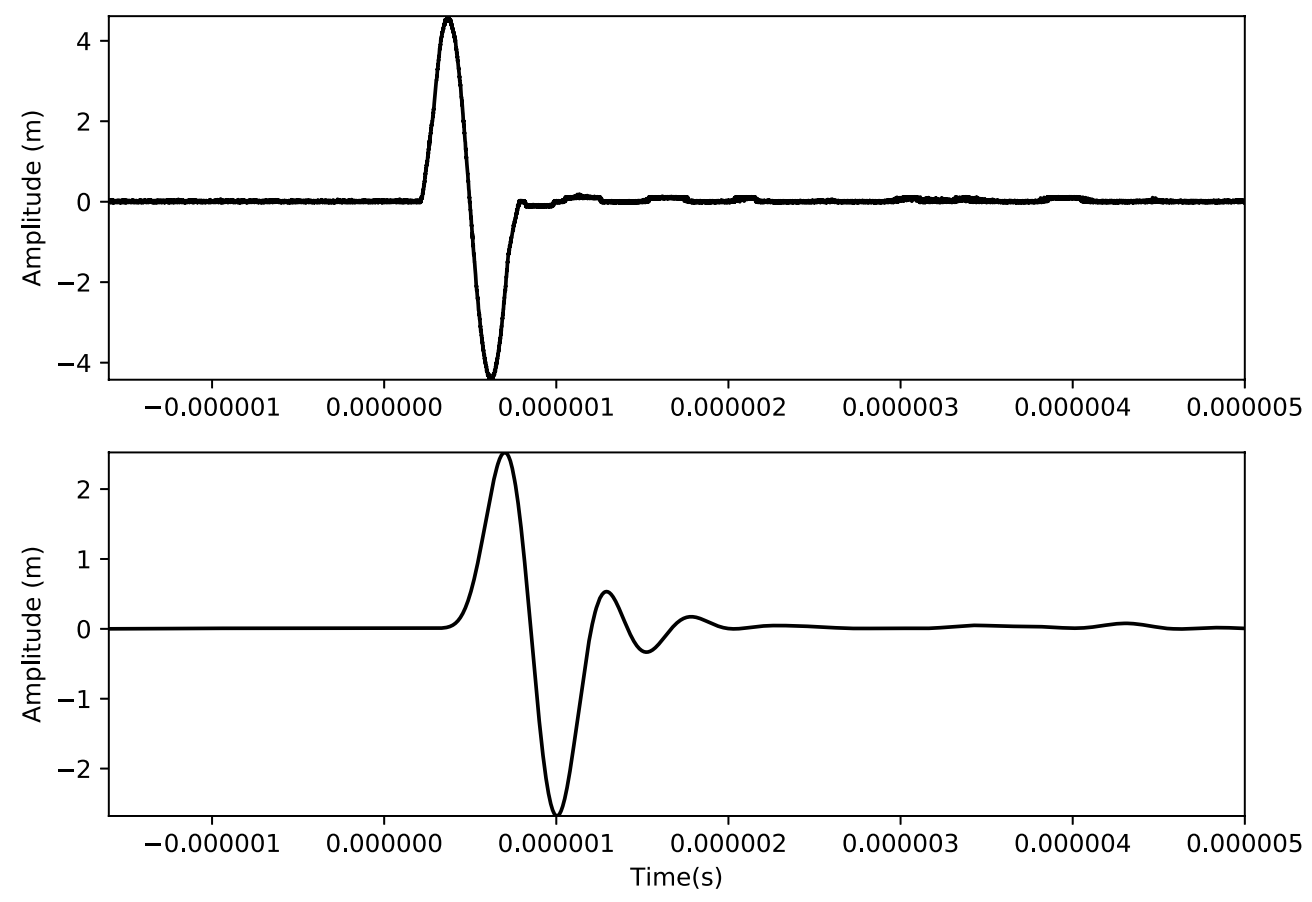

Figure B1. The input wavelet of the rock experiment (top) and the numerical modeling (bottom). In order to avoid the noise of the experimental wavelet, we use the filtered wavelet as the input.

\section{Data Availability Statement}

Data is available through Zaima and Katayama (2018), https://doi.org/10.1029/2018JB016377.

Acknowledgments

This research is a part of the $\mathrm{PhD}$ thesis of Ssu-Ting Lai, which is co-funded by French-National Program MOPGA (Make Our Planet Great Again) and Université de Paris, Institut de physique du globe de Paris. Numerical computations were partly performed on the S-CAPAD platform, IPGP, France. The authors would like to thank the constructive and fruitful reviews of Editor Y. Bernabe, Associate Editor, G. Rossi, J. Sarout, A. Nicolas and anonymous reviewers.

\section{References}

Audet, P., Bostock, M. G., Christensen, N. I., \& Peacock, S. M. (2009). Seismic evidence for overpressured subducted oceanic crust and megathrust fault sealing. Nature, 457(7225), 76-78. https://doi.org/10.1038/nature07650

Benson, P., Schubnel, A., Vinciguerra, S., Trovato, C., Meredith, P., \& Young, R. P. (2006). Modeling the permeability evolution of microcracked rocks from elastic wave velocity inversion at elevated isostatic pressure. Journal of Geophysical Research, 111, B04202. https:// doi.org/10.1029/2005JB003710

Bonner, B. P. (1974). Shear wave birefringence in dilating granite. Geophysical Research Letters, 1(5), 217-220. https://doi.org/10.1029/ GL001i005p00217

Brantut, N. (2018). Time-resolved tomography using acoustic emissions in the laboratory, and application to sandstone compaction. Geophysical Journal International, 213(3), 2177-2192. https://doi.org/10.1093/gji/ggy068

Capdeville, Y., Vilotte, J. P., \& Montagner, J. P. (2003). Coupling the spectral element method with a modal solution for elastic wave propagation in global earth models. Geophysical Journal International, 152(1), 34-67. https://doi.org/10.1046/j.1365-246X.2003.01808.x

Carcione, J. M., Kosloff, D., \& Kosloff, R. (1988). Wave propagation simulation in a linear viscoacoustic medium. Geophysical Journal, 93(2), 393-401. https://doi.org/10.1111/j.1365-246X.1988.tb02010.x

Chaljub, E., Komatitsch, D., Vilotte, J.-P., Capdeville, Y., Valette, B., \& Festa, G. (2007). Spectral-element analysis in seismology. Advances in Geophysics, 48, 365-419. https://doi.org/10.1016/S0065-2687(06)48007-9

Chaljub, E., Maufroy, E., Moczo, P., Kristek, J., Hollender, F., Bard, P.-Y., et al. (2015). 3-D numerical simulations of earthquake ground motion in sedimentary basins: Testing accuracy through stringent models. Geophysical Journal International, 201, 90-111. https://doi. org/10.1093/gii/ggu472

Christensen, N. I. (1984). Pore pressure and oceanic crustal seismic structure. Geophysical Journal International, 79(2), 411-423. https:// doi.org/10.1111/j.1365-246X.1984.tb02232.x

Cupillard, P., Delavaud, E., Burgos, G., Festa, G., Vilotte, J.-P., Capdeville, Y., \& Montagner, J.-P. (2012). RegSEM: A versatile code based on the Spectral Element Method to compute seismic wave propagation at the regional scale. Geophysical Journal International, 188, 1203-1220. https://doi.org/10.1111/j.1365-246X.2011.05311.x

De Basabe, J., \& Sen, M. (2014). Geophysical Journal International A comparison of finite-difference and spectral-element methods for elastic wave propagation in media with a fluid-solid interface. Geophysical Journal International, 200, 278-298. https://doi.org/10.1093/ gji/ggu389

Delavaud, É. (2007). Simulation numérique de la propagation d'ondes en milieu géologique complexe: Application à l'évaluation de la réponse sismique du bassin de caracas (Venezuela). PhD thesis. Institut de Physique du Globe de Paris. in French.

Di Carli, S., François-Holden, C., Peyrat, S., \& Madariaga, R. (2010). Dynamic inversion of the 2000 Tottori earthquake based on elliptical subfault approximations. Journal of Geophysical Research: Solid Earth, 115, B12328. https://doi.org/10.1029/2009JB006358 
Emmerich, H., \& Korn, M. (1987). Incorporation of attenuation into time-domain computations of seismic wave fields. Geophysics, 52(9), 1252-1264. https://doi.org/10.1190/1.1442386

Fliss, S., Bhat, H. S., Dmowska, R., \& Rice, J. R. (2005). Fault branching and rupture directivity. Journal of Geophysical Research, 110, B06312. https://doi.org/10.1029/2004JB003368

Fuji, N., Jang, H., Nakao, A., Kim, Y., Fernández-Blanco, D., Lee, S.-M., et al. (2021). A Possible roll-over slab geometry under the Caroline Plate Imaged by Monte Carlo finite-frequency traveltime inversion of teleseismic SS phases. Frontiers in Earth Science, 9, 29. https:// doi.org/10.3389/feart.2021.593947

Gabriel, A.-A., Ampuero, J.-P., Dalguer, L. A., \& Mai, P. M. (2012). The transition of dynamic rupture styles in elastic media under velocity-weakening friction. Journal of Geophysical Research, 117, B09311. https://doi.org/10.1029/2012JB009468

Geller, R. J., \& Ohminato, T. (1994). Computation of synthetic seismograms and their partial derivatives for heterogeneous media with arbitrary natural boundary conditions using the Direct Solution Method. Geophysical Journal International, 116(2), 421-446. https:// doi.org/10.1111/j.1365-246X.1994.tb01807.x

Gordon, R. B., \& Davis, L. A. (1968). Velocity and attenuation of seismic waves in imperfectly elastic rock. Journal of Geophysical Research, 73(12), 3917-3935. https://doi.org/10.1029/JB073i012p03917

Guéguen, Y., \& Schubnel, A. (2003). Elastic wave velocities and permeability of cracked rocks. Tectonophysics, 370(1), 163-176. https://doi. org/10.1016/S0040-1951(03)00184-7

Igel, H., Jahnke, G., \& Ben-Zion, Y. (2002). Numerical simulation of fault zone guided waves: Accuracy and 3-D effects. In M. Matsu'ura, P. Mora, A. Donnellan, \& X.-C. Yin (Eds.), Earthquake processes: Physical modelling, numerical simulation and data analysis part I (pp. 2067-2083): Birkhäuser Basel. https://doi.org/10.1007/978-3-0348-8203-3_11

Johnston, D. H., Toksöz, M. N., \& Timur, A. (1979). Attenuation of seismic waves in dry and saturated rocks: II. Mechanisms. Geophysics, 44(4), 691-711. https://doi.org/10.1190/1.1440970

Kjartansson, E. (1979). Constant Q-wave propagation and attenuation. Journal of Geophysical Research, 84(B9), 4737-4748. https://doi. org/10.1029/JB084iB09p04737

Komatitsch, D., \& Vilotte, J.-P. (1998). The Spectral Element method: An efficient tool to simulate the seismic response of 2D and 3D geological structures. Bulletin of the Seismological Society of America, 88, 368-392.

Kovalyshen, Y., Sarout, J., Dautriat, J., Maney, B., \& Lebedev, M. (2020). Ultrasonic characterization of anisotropic rocks: Impact of transducers' size and geometry on data interpretation. Geophysics, 85(4), C99-C105. https://doi.org/10.1190/geo2019-0083.1

Kovalyshen, Y., Sarout, J., \& Lebedev, M. (2018). On the interpretation of ultrasonic laboratory measurements in anisotropic media. Geophysics, 83(4), C173-C178. https://doi.org/10.1190/geo2017-0634.1

Kudo, Y., Sano, O., Murashige, N., Mizuta, Y., \& Nakagawa, K. (1992). Stress-induced crack path in Aji granite under tensile stress. Pure and Applied Geophysics, 138(4), 641-656. https://doi.org/10.1007/BF00876342

Lai, S.-T., Fuji, N., \& Katayama, I. (2019). Time-lapse elastic properties of cracked granite during deformation inferred from laboratory experiments using 2D waveform modelling. The 81st EAGE conference and exhibition. (pp. 1-5). https://doi.org/10.3997/2214-4609.201900662

Lay, T., \& Wallace, T. (1995). Modern global Seimology: Academic.

Lee, S.-J., Huang, B.-S., Ando, M., Chiu, H.-C., \& Wang, J.-H. (2011). Evidence of large scale repeating slip during the 2011 Tohoku-Oki earthquake. Geophysical Research Letters, 38(19), L19306. https://doi.org/10.1029/2011GL049580

Liu, H.-P., Anderson, D. L., \& Kanamori, H. (1976). Velocity dispersion due to anelasticity; implications for seismology and mantle composition. Geophysical Journal International, 47(1), 41-58. https://doi.org/10.1111/j.1365-246X.1976.tb01261.x

Lockner, D. A., Walsh, J. B., \& Byerlee, J. D. (1977). Changes in seismic velocity and attenuation during deformation of granite. Journal of Geophysical Research, 82(33), 5374-5378. https://doi.org/10.1029/JB082i033p05374

Moczo, P., \& Kristek, J. (2005). On the rheological models used for time-domain methods of seismic wave propagation. Geophysical Research Letters, 32(1), L01306. https://doi.org/10.1029/2004GL021598

Möllhoff, M., Bean, C. J., \& Meredith, P. G. (2010). Rock fracture compliance derived from time delays of elastic waves. Geophysical Prospecting, 58(6), 1111-1122. https://doi.org/10.1111/j.1365-2478.2010.00887.x

Pageot, D., Leparoux, D., Le Feuvre, M., Durand, O., Côte, P., \& Capdeville, Y. (2017). Improving the seismic small-scale modelling by comparison with numerical methods. Geophysical Journal International, 211(1), 637-649. https://doi.org/10.1093/gii/ggx309

Peacock, S., Christensen, N., Bostock, M., \& Audet, P. (2011). High pore pressures and porosity at $35 \mathrm{~km}$ depth in the Cascadia subduction zone. Geology, 39, 471-474. https://doi.org/10.1130/G31649.1

Peter, D., Komatitsch, D., Luo, Y., Martin, R., Le Goff, N., Casarotti, E., et al. (2011). Forward and adjoint simulations of seismic wave propagation on fully unstructured hexahedral meshes. Geophysical Journal International, 186(2), 721-739. https://doi. $\operatorname{org} / 10.1111 / j .1365-246 X .2011 .05044 . x$

Properties Silicone Rubber. (2020). Retrieved from 9 September 2020. https://www.azom.com/properties.aspx?ArticleID=920

Sarout, J., Cazes, E., Delle Piane, C., Arena, A., \& Esteban, L. (2017). Stress-dependent permeability and wave dispersion in tight cracked rocks: Experimental validation of simple effective medium models. Journal of Geophysical Research: Solid Earth, 122, 6180-6201. https://doi.org/10.1002/2017JB014147

Scholz, C. H., Sykes, L. R., \& Aggarwal, Y. P. (1973). Earthquake prediction: A physical basis. Science, 181(4102), 803-810. https://doi. org $/ 10.1126 /$ science.181.4102.803

Simmons, G., \& Brace, W. F. (1965). Comparison of static and dynamic measurements of compressibility of rocks. Journal of Geophysical Research, 70(22), 5649-5656. https://doi.org/10.1029/JZ070i022p05649

Solymosi, B., Favretto-Cristini, N., Monteiller, V., Komatitsch, D., Cristini, P., Arntsen, B., \& Ursin, B. (2018). How to adapt numerical simulation of wave propagation and ultrasonic laboratory experiments to be comparable-A case study for a complex topographic model. Geophysics, 83, T195-T207. https://doi.org/10.1190/geo2017-0536.1

Tisato, N., \& Quintal, B. (2014). Laboratory measurements of seismic attenuation in sandstone: Strain versus fluid saturation effects. Geophysics, 79(5), WB9-WB14. https://doi.org/10.1190/geo2013-0419.1

Toksöz, M. N., Johnston, D. H., \& Timur, A. (1979). Attenuation of seismic waves in dry and saturated rocks: I. Laboratory measurements. Geophysics, 44(4), 681-690. https://doi.org/10.1190/1.1440969

Walsh, J. B. (1965). The effect of cracks on the compressibility of rock. Journal of Geophysical Research, 70(2), 381-389. https://doi. org/10.1029/JZ070i002p00381

Walsh, J. B. (1966). Seismic wave attenuation in rock due to friction. Journal of Geophysical Research, 71(10), 2591-2599. https://doi. org/10.1029/JZ071i010p02591

Wang, X.-Q., Schubnel, A., Fortin, J., David, E. C., Guéguen, Y., \& Ge, H.-K. (2012). High Vp/Vs ratio: Saturated cracks or anisotropy effects? Geophysical Research Letters, 39(11), L11307. https://doi.org/10.1029/2012GL051742 
Watanabe, T., \& Higuchi, A. (2015). Simultaneous measurements of elastic wave velocities and electrical conductivity in a brine-saturated granitic rock under confining pressures and their implication for interpretation of geophysical observations. Progress in Earth and Planetary Science, 2, 37. https://doi.org/10.1186/s40645-015-0067-0

Winkler, K. W., \& Nur, A. (1982). Seismic attenuation: Effects of pore fluids and frictional-sliding. Geophysics, 47(1), 1-15. https://doi. org/10.1190/1.1441276

Yoshimitsu, N., Furumura, T., \& Maeda, T. (2016). Geometric effect on a laboratory-scale wavefield inferred from a three-dimensional numerical simulation. Journal of Applied Geophysics, 132, 184-192. https://doi.org/10.1016/j.jappgeo.2016.07.002

Zaima, K., \& Katayama, I. (2018). Evolution of elastic wave velocities and amplitudes during triaxial deformation of Aji granite under dry and water-saturated conditions. Journal of Geophysical Research: Solid Earth, 123(11), 9601-9614. https://doi.org/10.1029/2018JB016377

Zotz-Wilson, R., Douma, L. A. N. R., Sarout, J., Dautriat, J., Dewhurst, D., \& Barnhoorn, A. (2020). Ultrasonic imaging of the onset and growth of fractures within partially saturated whitby mudstone using coda wave decorrelation inversion. Journal of Geophysical Research: Solid Earth, 125, e2020JB020042. https://doi.org/10.1029/2020JB020042 